\title{
REVISITING SCALING RELATIONS FOR GIANT RADIO HALOS IN GALAXY CLUSTERS
}

\author{
R. Cassano ${ }^{1}$, S. Ettori ${ }^{2,3}$, G. Brunetti ${ }^{1}$, S. Giacintucci ${ }^{4,5}$, G. W. Pratt $^{6}$, T. Venturi ${ }^{1}$,

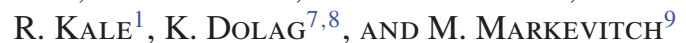 \\ ${ }^{1}$ INAF/IRA, via Gobetti 101, I-40129 Bologna, Italy \\ 2 INAF/Osservatorio Astronomico di Bologna, via Ranzani 1, I-40127 Bologna, Italy \\ 3 INFN, Sezione di Bologna, viale Berti Pichat 6/2, I-40127 Bologna, Italy \\ ${ }^{4}$ Department of Astronomy, University of Maryland, College Park, MD 20742-2421, USA \\ 5 Joint Space-Science Institute, University of Maryland, College Park, MD 20742-2421, USA \\ ${ }^{6}$ Laboratoire AIM, IRFU/Service dAstrophysique-CEA/DSM-CNRS-Université Paris Diderot, Bât. 709, \\ CEA-Saclay, F-91191 Gif-sur-Yvette Cedex, France \\ ${ }^{7}$ University Observatory Munich, Scheinerstr. 1, D-81679 Munich, Germany \\ ${ }^{8}$ Max-Planck-Institut für Astrophysik, Karl-Schwarzschild Strasse 1, Garching bei München, Germany \\ ${ }^{9}$ Astrophysics Science Division, NASA/Goddard Space Flight Center, Greenbelt, MD 20771, USA \\ Received 2013 June 14; accepted 2013 September 10; published 2013 October 23
}

\begin{abstract}
Many galaxy clusters host megaparsec-scale radio halos, generated by ultrarelativistic electrons in the magnetized intracluster medium. Correlations between the synchrotron power of radio halos and the thermal properties of the hosting clusters were established in the last decade, including the connection between the presence of a halo and cluster mergers. The X-ray luminosity and redshift-limited Extended GMRT Radio Halo Survey provides a rich and unique dataset for statistical studies of the halos. We uniformly analyze the radio and X-ray data for the GMRT cluster sample, and use the new Planck Sunyaev-Zel'dovich (SZ) catalog to revisit the correlations between the power of radio halos and the thermal properties of galaxy clusters. We find that the radio power at $1.4 \mathrm{GHz}$ scales with the cluster X-ray $(0.1-2.4 \mathrm{keV})$ luminosity computed within $R_{500}$ as $P_{1.4} \sim L_{500}^{2.1 \pm 0.2}$. Our bigger and more homogenous sample confirms that the X-ray luminous $\left(L_{500}>5 \times 10^{44} \mathrm{erg} \mathrm{s}^{-1}\right)$ clusters branch into two populations - radio halos lie on the correlation, while clusters without radio halos have their radio upper limits well below that correlation. This bimodality remains if we excise cool cores from the X-ray luminosities. We also find that $P_{1.4}$ scales with the cluster integrated SZ signal within $R_{500}$, measured by Planck, as $P_{1.4} \sim Y_{500}^{2.05 \pm 0.28}$, in line with previous findings. However, contrary to previous studies that were limited by incompleteness and small sample size, we find that "SZ-luminous" $Y_{500}>6 \times 10^{-5} \mathrm{Mpc}^{2}$ clusters show a bimodal behavior for the presence of radio halos, similar to that in the radio-X-ray diagram. Bimodality of both correlations can be traced to clusters dynamics, with radio halos found exclusively in merging clusters. These results confirm the key role of mergers for the origin of giant radio halos, suggesting that they trigger the relativistic particle acceleration.
\end{abstract}

Key words: galaxies: clusters: general - radiation mechanisms: non-thermal - radio continuum: general X-rays: galaxies: clusters

Online-only material: color figures

\section{INTRODUCTION}

The presence of non-thermal components (relativistic particles and magnetic fields) mixed with the thermal intracluster medium (ICM) has been revealed by radio observations of galaxy clusters showing diffuse, giant Mpc-scale synchrotron radio halos (RHs) and radio relics in a substantial fraction of massive clusters (e.g., Ferrari et al. 2008; Cassano 2009; Feretti et al. 2012 for reviews).

Giant RHs are the most spectacular and best studied clusterscale non-thermal sources. Their origin is still poorly understood. One possibility is synchrotron emission from secondary electrons generated by the collisions between cosmic ray protons and thermal protons (so-called "secondary models," e.g., Dennison 1980). However, the same collisions should produce gamma rays through the generation and decay of neutral pions. The non-detection of nearby galaxy clusters in the $\gamma$-ray band at $0.1-100 \mathrm{GeV}$ puts serious limits on the contribution of secondary electrons to the RH emission (Ackermann et al. 2010; Jeltema \& Profumo 2011; Brunetti et al. 2012).

A second possibility is that the turbulence, generated in the ICM during cluster-cluster mergers, re-accelerates preexisting GeV electrons (e.g., Brunetti et al. 2001; Petrosian 2001). The "historical" motivation for turbulent acceleration for the origin of RHs comes from the high-frequency steepening of the Coma halo spectrum, implying that the mechanism responsible for the acceleration of the emitting electrons is inefficient (e.g., Schlickeiser et al. 1987). More recently, the discovery of RHs with extremely steep spectra, ${ }^{10} \alpha \sim 1.5-2$, support turbulent re-acceleration and disfavor a "secondary" origin of giant RHs (e.g., Brunetti et al. 2008; Dallacasa et al. 2009; Giovannini et al. 2009; Macario et al. 2010, 2011; Giacintucci et al. 2011, 2013; Bonafede et al. 2012; Venturi et al. 2013).

Studies of statistical properties of giant RHs in clusters and their connection with the cluster dynamics are extremely useful to constrain the origin of halos. From the inspection of the NRAO VLA Sky Survey (NVSS; Condon et al. 1998) fields containing X-ray selected galaxy clusters, Giovannini et al. (1999) concluded that RHs are rare at low X-ray luminosities $\left(L_{X} \lesssim 10^{45} h_{50}^{-2} \mathrm{erg} \mathrm{s}^{-1}\right.$ ), while only the most X-ray luminous systems host RHs, with a probability of $\sim 1 / 3$. Since then, a number of correlations have been found between thermal and non-thermal cluster properties, suggesting a tight connection between them. In particular, the synchrotron monochromatic

\footnotetext{
$\overline{10}$ Here, we adopt the convention $f_{v} \propto v^{-\alpha}$.
} 
radio power of halos at $1.4 \mathrm{GHz}\left(P_{1.4}\right)$ has been found to increase with the cluster X-ray luminosity, temperature, and total mass (e.g., Colafrancesco 1999; Liang 1999; Feretti 2002, 2003; Govoni et al. 2001; Enßlin \& Röttgering 2002; Cassano et al. 2006). These scalings call into question the rarity of halos in clusters of low X-ray luminosity, suggesting that the lack of RH detections in those clusters may result from the combination of the radio power-X-ray luminosity correlation and the sensitivity of the radio survey (e.g., Kempner \& Sarazin 2001).

There is also substantial evidence that RHs are found in clusters with significant substructure in the X-ray images, as well as complex gas temperature distribution, which are signatures of cluster mergers (e.g., Schuecker et al. 2001; Govoni et al. 2004; Markevitch \& Vikhlinin 2001). In particular, Buote (2001) provided the first quantitative comparison of the dynamical state of clusters with $\mathrm{RH}$, discovering a correlation between the RH luminosity at $1.4 \mathrm{GHz}$ and the magnitude of the dipole power ratio $P_{1} / P_{0}$, which is a measure of the cluster's $\mathrm{X}$-ray morphological disturbance. However, these observational claims were based on collections of data from the literature and not on statistical samples of galaxy clusters.

An important step was recently obtained through deep radio observations of a complete sample of galaxy clusters as part of the Giant Metrewave Radio Telescope (GMRT) RH Survey (GRHS hereafter; Venturi et al. 2007, 2008). These observations confirmed that $\mathrm{RH}$ are not ubiquitous in clusters. They are found only in $\sim 30 \%$ of the X-ray luminous systems $\left(L_{X}(0.1-2.4 \mathrm{keV}) \geqslant 5 \times 10^{44} \mathrm{erg} \mathrm{s}^{-1}\right)$. The sensitivity reached by these observations allowed for the first time to place deep upper limits on the diffuse radio flux of clusters without giant $\mathrm{RH}$ and to show that clusters branch into two populations: RHs trace the correlation between $P_{1.4}$ and $L_{X}$, while the upper limits on the radio luminosity of clusters with no RH lie about one order of magnitude below that correlation (e.g., Brunetti et al. 2007, 2009). Using several methods to characterize cluster substructures, it was also shown that clusters with and without RH can be quantitatively differentiated in terms of their dynamical properties, with RHs always associated with dynamically disturbed clusters while clusters without RHs are more "relaxed" (Cassano et al. 2010).

Sensitivity is critical in these studies. Indeed, analyses based on all-sky surveys, such as the NVSS and WENSS that have a sensitivity four to five times worse than the GRHS, do not allow to recover a bimodal behavior in the radio-X-ray diagram (e.g., Rudnick \& Lemmerman 2009 for the WENSS). On the other hand, evidence for a bimodal behavior of clusters was recently found through a stacking analysis of clusters in the SUMSS (Brown et al. 2011).

More recently, Basu (2012) cross-correlated the Planck ESZ cluster catalog (Planck Collaboration et al. 2011a) with radio data from the GRHS. He found a correlation between $P_{1.4}$ and the integrated Sunyaev-Zel'dovich (SZ) effect measurements, but did not find a strong indication for a bimodal split between $\mathrm{RH}$ and radio-quiet clusters. To explain this apparent lack of bimodality in SZ, Basu (2012) suggested that X-ray observations could be biased towards the detection of low-mass cool-core clusters, whereas SZ selection picks up the most massive systems, irrespective of their dynamical states.

In this paper, we improve on the previous statistical studies on the distribution of clusters in the $P_{1.4}-L_{X}$ diagram by using $\mathrm{RH}$ and clusters with radio upper limits from the GRHS and its extension and, when needed, including RHs from the literature. Contrary to previous analyses that used cluster X-ray and radio halo luminosities from the literature, we reevaluate the radio and X-ray luminosities in a homogeneous way. In particular, we derive the X-ray luminosity within $R_{500}{ }^{11}$ and include the correction due to the contribution of the cool core (when present). Furthermore, since the integrated SZ signal is a more robust indicator of the cluster mass than the X-ray luminosity (e.g., Motl et al. 2005; Nagai 2006), we cross-checked our sample with the recent Planck SZ (PSZ) cluster catalog (Planck Collaboration et al. 2013b) and derived the distribution of clusters in the radio-SZ plane.

In Section 2, we describe the cluster sample. In Section 3, we summarize the procedure to derive different cluster quantities (radio-halo power, X-ray luminosity, SZ flux, morphological parameters), identify cool-core clusters in the sample, and fit the scaling relations. In Section 4, we report on the expected theoretical scalings. We derive the distribution of clusters in the radio-X-ray diagrams in Section 5, and in the radio-SZ (mass) diagrams in Section 6. Finally, in Section 7, we give a summary and conclusions.

A $\Lambda$ CDM cosmology $\left(H_{o}=70 \mathrm{~km} \mathrm{~s}^{-1} \mathrm{Mpc}^{-1}, \Omega_{m}=0.3\right.$, $\left.\Omega_{\Lambda}=0.7\right)$ is adopted.

\section{THE SAMPLE}

The GRHS is a deep, pointed radio survey of clusters selected from the ROSAT-ESO Flux Limited X-ray (REFLEX; Böhringer et al. 2004) and extended ROSAT Brightest Cluster Sample (eBCS; Ebeling et al. 1998, 2000) catalogs. These two catalogs have almost the same flux limit in the $0.1-2.4 \mathrm{keV}$ band $\left(\gtrsim 3 \times 10^{-12} \mathrm{erg} \mathrm{s}^{-1} \mathrm{~cm}^{-2}\right)$ and their combination yields a homogeneous, flux-limited sample of clusters. The GRHS consists of 50 galaxy clusters with $z=0.2-0.4$, X-ray luminosity $L_{X}>5 \times 10^{44} \mathrm{erg} \mathrm{s}^{-1}$, and declination $\delta \geqslant 30^{\circ}$ for the REFLEX sample and $15^{\circ} \leqslant \delta \leqslant 60^{\circ}$ for the eBCS sample. With the above selection criteria, the sample is X-ray luminositylimited up to $z \simeq 0.25$ and X-ray flux-limited at higher redshift ${ }^{12}$ (see Figures 1 and 2 in Cassano et al. 2008).

Recently, we have undertaken an extension of the GRHS by considering all clusters in the REFLEX and eBCs catalogs with $\delta>-30^{\circ}$ with the same $z$ and $L_{X}$ selection (Kale et al. 2013). This extension yields a final sample of 67 galaxy clusters, which we refer to as the extended GMRT RH Survey (EGRHS). For all clusters in the EGRHS with the radio data already available, we searched the ROSAT and Chandra archive and found data for a subsample of 40 galaxy clusters:

1. 29 with radio upper limits;

2. 8 with giant radio halos;

3. 3 with radio halos with ultra-steep spectra. ${ }^{13}$

In addition to clusters belonging to the EGRHS, we also searched in the X-ray archive and found data for 14 clusters with RHs from the literature:

1. 11 with giant radio halos;

2. 3 with radio halos with ultra-steep spectra.

The total sample with radio and X-ray information consists of 54 galaxy clusters, whose main properties are reported in Table 1:

\footnotetext{
$11 R_{500}$ is the radius corresponding to a total density contrast $500 \rho_{c}(z)$, where $\rho_{c}(z)$ is the critical density of the universe at the cluster redshift.

12 This implies a minimum $L_{X} \sim 10^{45} \mathrm{erg} \mathrm{s}^{-1}$ at $z \sim 0.35$.

13 We do not include a fourth one, RXCJ1514.9-1523 (Giacintucci et al. 2011), which was only recently observed by Chandra, the data analysis is still ongoing (S. Giacintucci et al., in preparation).
} 
Table 1

Cluster's Properties

\begin{tabular}{|c|c|c|c|c|c|c|c|c|c|}
\hline Cluster Name & R.A.J2000 & Decl.J2000 $_{\text {J }}$ & $z$ & $L_{500}$ & $L_{500, \text { cor }}$ & $L_{\text {core }} / L_{500}$ & $P_{1.4}$ & X-Ray & SZ \\
\hline \multicolumn{10}{|l|}{ Upper limits (EGRHS) } \\
\hline A2697 & 000311.8 & -060510 & 0.232 & $7.29 \pm 0.41$ & $7.29 \pm 0.41$ & 0.34 & $<0.41^{v}$ & $19(\mathrm{H})$ & $\sqrt{ }$ \\
\hline A141 & 010534.8 & -243917 & 0.230 & $6.82 \pm 0.27$ & $6.82 \pm 0.27$ & 0.13 & $<0.43^{v}$ & $33(\mathrm{H})$ & $\sqrt{ }$ \\
\hline A3088 & 030704.1 & -284014 & 0.254 & $6.97 \pm 0.09$ & $5.63 \pm 0.08$ & 0.46 & $<0.43^{v}$ & $19(\mathrm{C})$ & $\sqrt{ }$ \\
\hline RXCJ0437.1 + 0043 & 043710.1 & +004338 & 0.284 & $6.99 \pm 0.08$ & $6.15 \pm 0.08$ & 0.45 & $<0.65^{k}$ & $30(\mathrm{C})$ & \\
\hline RXCJ1115.8+ 0129 & 111554.0 & +012944 & 0.350 & $12.69 \pm 0.11$ & $8.21 \pm 0.10$ & $0.63^{c c}$ & $<0.47^{v}$ & $39(\mathrm{C})$ & $\sqrt{ }$ \\
\hline A2485 & 224832.9 & -160623 & 0.247 & $3.27 \pm 0.07$ & $3.07 \pm 0.07$ & 0.39 & $<0.47^{k}$ & $20(\mathrm{C})$ & \\
\hline A2631 & 233740.6 & +001636 & 0.278 & $8.62 \pm 0.70$ & $8.62 \pm 0.70$ & 0.21 & $<0.41^{v}$ & $15(\mathrm{H})$ & $\sqrt{ }$ \\
\hline A2645 & 234116.8 & -090139 & 0.251 & $4.13 \pm 0.4$ & $4.13 \pm 0.4$ & 0.43 & $<0.59^{q}$ & $35(\mathrm{H})$ & $\sqrt{ }$ \\
\hline A2667 & 235140.7 & -260501 & 0.226 & $12.50 \pm 0.4$ & $10.94 \pm 0.4$ & $0.46^{c c}$ & $<0.45^{v}$ & $21(\mathrm{H})$ & $\sqrt{ }$ \\
\hline Z348 & 010650.3 & +010317 & 0.255 & $6.30 \pm 0.60$ & $4.26 \pm 0.60$ & $0.54^{c c}$ & $<0.65^{k}$ & $13(\mathrm{H})$ & \\
\hline RXJ0142.0 + 2131 & 014203.1 & +213039 & 0.280 & $6.00 \pm 0.10$ & $6.00 \pm 0.10$ & 0.34 & $<0.45^{k}$ & $20(\mathrm{C})$ & $\sqrt{ }$ \\
\hline A267 & 015252.2 & +010246 & 0.230 & $6.29 \pm 0.44$ & $5.94 \pm 0.44$ & 0.36 & $<0.34^{k}$ & $16(\mathrm{H})$ & $\sqrt{ }$ \\
\hline RXJ0439.0 + 0715 & 043901.2 & +071536 & 0.244 & $8.05 \pm 0.59$ & $7.69 \pm 0.58$ & 0.36 & $<0.46^{k}$ & $19(\mathrm{H})$ & $\sqrt{ }$ \\
\hline RXJ0439.0+0520 & 043902.2 & +052043 & 0.208 & $5.35 \pm 0.47$ & $4.05 \pm 0.46$ & $0.55^{c c}$ & $<0.32^{k}$ & $12(\mathrm{H})$ & \\
\hline A611 & 080058.1 & +360441 & 0.288 & $4.96 \pm 0.64$ & $4.96 \pm 0.64$ & 0.46 & $<0.43^{v}$ & $17(\mathrm{H})$ & $\sqrt{ }$ \\
\hline Z2089 & 090045.9 & +205513 & 0.235 & $5.28 \pm 0.41$ & $3.98 \pm 0.41$ & $0.52^{c c}$ & $<0.26^{v}$ & $17(\mathrm{H})$ & \\
\hline A781 & 092023.2 & +302615 & 0.298 & $5.44 \pm 0.14$ & $5.44 \pm 0.14$ & 0.12 & $<0.36^{v}$ & $10(\mathrm{C})$ & $\sqrt{ }$ \\
\hline Z2701 & 095255.3 & +515252 & 0.214 & $4.72 \pm 0.43$ & $3.38 \pm 0.42$ & $0.55^{c c}$ & $<0.35^{v}$ & $10(\mathrm{H})$ & \\
\hline A1423 & 115722.5 & +333918 & 0.213 & $5.35 \pm 0.37$ & $4.76 \pm 0.38$ & 0.41 & $<0.38^{v}$ & $19(\mathrm{H})$ & $\sqrt{ }$ \\
\hline A1576 & 123649.1 & +631130 & 0.30 & $6.68 \pm 0.14$ & $6.38 \pm 0.14$ & 0.24 & $<0.64^{k}$ & $17(\mathrm{P})$ & $\sqrt{ }$ \\
\hline RXJ1532.9+3021 & 153254.2 & +302111 & 0.345 & $17.94 \pm 0.27$ & $12.28 \pm 0.22$ & $0.64^{c c}$ & $<0.66^{v}$ & $22(\mathrm{C})$ & \\
\hline A2146 & 155604.7 & +662024 & 0.234 & $5.69 \pm 0.04$ & $5.69 \pm 0.04$ & 0.42 & $<0.39^{r}$ & $65(\mathrm{C})$ & $\sqrt{ }$ \\
\hline A2261 & 172228.3 & +320913 & 0.224 & $8.98 \pm 0.38$ & $7.79 \pm 0.37$ & 0.43 & $<0.32^{k}$ & $30(\mathrm{H})$ & $\sqrt{ }$ \\
\hline RXJ2228.6+2037 & 222834.4 & +203647 & 0.418 & $11.71 \pm 0.20$ & $11.71 \pm 0.20$ & 0.29 & $<0.95^{v}$ & $20(\mathrm{C})$ & $\sqrt{ }$ \\
\hline A2537 & 230823.3 & -021131 & 0.297 & $5.48 \pm 0.14$ & $4.54 \pm 0.07$ & 0.45 & $<0.51^{v}$ & $39(\mathrm{C})$ & $\sqrt{ }$ \\
\hline RXJ0027.6+2616 & 002749.8 & +261626 & 0.365 & $3.52 \pm 0.11$ & $3.52 \pm 0.11$ & 0.24 & $<0.74^{v}$ & $22(\mathrm{C})$ & \\
\hline Z5699 & 130600.0 & +263058 & 0.306 & $4.74 \pm 0.08$ & $4.74 \pm 0.08$ & 0.18 & $<0.59^{v}$ & $26(\mathrm{C})$ & \\
\hline Z5768 & 131131.5 & +220005 & 0.266 & $1.66 \pm 0.06$ & $1.66 \pm 0.06$ & 0.10 & $<0.36^{v}$ & $27(\mathrm{C})$ & \\
\hline S780 & 145929.3 & -181113 & 0.236 & $8.68 \pm 0.10$ & $6.32 \pm 0.05$ & 0.43 & $<0.38^{v}$ & $40(\mathrm{C})$ & $\sqrt{ }$ \\
\hline \multicolumn{10}{|l|}{ Radio Halos (EGRHS) } \\
\hline A2744 & 001418.8 & -302300 & 0.307 & $14.73 \pm 0.24$ & $14.73 \pm 0.24$ & 0.17 & $18.62 \pm 0.94^{\mathrm{a}}$ & $14(\mathrm{P})$ & $\sqrt{ }$ \\
\hline A0209 & 013153.0 & -133634 & 0.206 & $7.62 \pm 0.48$ & $7.62 \pm 0.48$ & 0.31 & $1.99 \pm 0.21^{\mathrm{a}}$ & $11(\mathrm{H})$ & $\sqrt{ }$ \\
\hline A2163 & 161546.9 & -060845 & 0.203 & $21.95 \pm 0.33$ & $21.95 \pm 0.33$ & 0.25 & $22.91 \pm 1.16^{\mathrm{a}}$ & $7(\mathrm{P})$ & $\sqrt{ }$ \\
\hline RXCJ2003.5-2323 & 200330.4 & -232305 & 0.317 & $9.17 \pm 0.09$ & $9.17 \pm 0.09$ & 0.09 & $10.71 \pm 1.73^{b}$ & $50(\mathrm{C})$ & $\sqrt{ }$ \\
\hline A520 & 045419.0 & +025649 & 0.203 & $7.81 \pm 0.21$ & $7.81 \pm 0.21$ & 0.18 & $2.45 \pm 0.18^{\mathrm{a}}$ & $5(\mathrm{P})$ & $\sqrt{ }$ \\
\hline A773 & 091759.4 & +514223 & 0.217 & $7.30 \pm 0.57$ & $7.30 \pm 0.57$ & 0.35 & $1.48 \pm 0.16^{\mathrm{a}}$ & $17(\mathrm{H})$ & $\sqrt{ }$ \\
\hline A1758a & 133232.1 & +503037 & 0.280 & $8.80 \pm 0.16$ & $8.80 \pm 0.16$ & 0.18 & $5.75 \pm 0.98^{\mathrm{a}}$ & $16(\mathrm{P})$ & $\sqrt{ }$ \\
\hline A2219 & 164021.1 & +464116 & 0.228 & $14.78 \pm 0.19$ & $14.78 \pm 0.19$ & 0.20 & $5.63 \pm 0.80^{\mathrm{a}}$ & $16(\mathrm{P})$ & $\sqrt{ }$ \\
\hline $\mathrm{A} 0521^{U}$ & 045409.1 & -101419 & 0.248 & $8.28 \pm 0.07$ & $8.28 \pm 0.07$ & 0.08 & $1.45 \pm 0.13^{\mathrm{i}}$ & $39(\mathrm{C})$ & $\sqrt{ }$ \\
\hline $\mathrm{A} 697^{U}$ & 084253.3 & +362012 & 0.282 & $13.04 \pm 0.61$ & $13.04 \pm 0.61$ & 0.33 & $1.51 \pm 0.14^{1}$ & $28(\mathrm{H})$ & $\sqrt{ }$ \\
\hline $\mathrm{A} 1300^{U}$ & 113156.3 & -195537 & 0.308 & $11.47 \pm 0.37$ & $11.47 \pm 0.37$ & 0.18 & $3.8 \pm 1.43^{\mathrm{p}}$ & $9(\mathrm{P})$ & $\sqrt{ }$ \\
\hline \multicolumn{10}{|l|}{ Radio Halos (literature) } \\
\hline CL0016+16 & 001833.3 & +162636 & 0.541 & $15.54 \pm 0.28$ & $15.54 \pm 0.28$ & 0.16 & $5.01 \pm 0.31^{\mathrm{a}}$ & $43(\mathrm{P})$ & $\sqrt{ }$ \\
\hline A1914 & 142603.0 & +374932 & 0.171 & $11.17 \pm 0.13$ & $10.25 \pm 0.13$ & 0.39 & $5.62 \pm 0.43^{\mathrm{a}}$ & $9(\mathrm{P})$ & $\sqrt{ }$ \\
\hline A665 & 083045.2 & +655255 & 0.182 & $8.36 \pm 0.09$ & $8.30 \pm 0.07$ & 0.22 & $2.51 \pm 0.21^{\mathrm{a}}$ & $38(\mathrm{P})$ & $\sqrt{ }$ \\
\hline A545 & 053220.2 & -113154 & 0.154 & $6.31 \pm 0.09$ & $6.31 \pm 0.09$ & 0.23 & $1.41 \pm 0.22^{\mathrm{a}}$ & $14(\mathrm{P})$ & $\sqrt{ }$ \\
\hline Coma & 125948.7 & +275850 & 0.023 & $3.39 \pm 0.03^{*}$ & $\ldots$ & & $0.76 \pm 0.06^{\mathrm{c}}$ & $\ldots$ & $\sqrt{ }$ \\
\hline A2256 & 170343.5 & +784303 & 0.058 & $4.44 \pm 0.02$ & $4.44 \pm 0.02$ & 0.22 & $0.85 \pm 0.08^{\mathrm{d}}$ & $17(\mathrm{P})$ & $\sqrt{ }$ \\
\hline Bullet & 065829.2 & -555710 & 0.296 & $22.54 \pm 0.52$ & $22.54 \pm 0.52$ & 0.22 & $23.44 \pm 1.51^{\mathrm{e}}$ & $5(\mathrm{P})$ & $\sqrt{ }$ \\
\hline A2255 & 171231.0 & +640533 & 0.081 & $3.31 \pm 0.03$ & $3.31 \pm 0.03$ & 0.14 & $0.81 \pm 0.17^{\mathrm{f}}$ & $15(\mathrm{P})$ & $\sqrt{ }$ \\
\hline A2319 & 192045.3 & +435743 & 0.056 & $7.96 \pm 0.08$ & $7.87 \pm 0.08$ & 0.31 & $2.45 \pm 0.19^{\mathrm{g}}$ & $3(\mathrm{P})$ & $\sqrt{ }$ \\
\hline MACS J0717.5+3745 & 071733.8 & +374520 & 0.548 & $24.05 \pm 0.22$ & $24.05 \pm 0.22$ & 0.17 & $52.48 \pm 20.56^{\mathrm{h}}$ & $60(\mathrm{C})$ & $\sqrt{ }$ \\
\hline A1995 & 145250.4 & +580248 & 0.319 & $6.03 \pm 0.08$ & $6.03 \pm 0.08$ & 0.43 & $1.66 \pm 0.23^{\mathrm{a}}$ & 48 (C) & $\sqrt{ }$ \\
\hline MACSJ1149.5+2223 & 114934.3 & +222342 & 0.544 & $15.50 \pm 0.29$ & $15.50 \pm 0.29$ & 0.18 & $2.29 \pm 0.95^{\mathrm{m}}$ & $19(\mathrm{C})$ & $\sqrt{ }$ \\
\hline PLCKG171.9-40.7 & 031257.4 & +082210 & 0.270 & $11.28 \pm 0.02^{* *}$ & & & $4.90 \pm 1.35^{\mathrm{n}}$ & $\ldots$ & 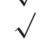 \\
\hline $\mathrm{A} 754^{U}$ & 090850.1 & -093812 & 0.054 & $4.75 \pm 0.033$ & $4.75 \pm 0.033$ & 0.23 & $0.63 \pm 0.07^{\circ}$ & $8(\mathrm{P})$ & $\sqrt{ }$ \\
\hline
\end{tabular}

Notes. The first part of the table contains clusters with radio upper limits belonging to the EGRHS (from Venturi et al. 2008; Kale et al. 2013); the second part contains clusters with giant RHs belonging to the EGRHS; and the third part contains clusters with giant RHs not belonging to the EGRHS; clusters marked with ${ }^{U}$ are those hosting USSRH $(\alpha>1.5$ ). Columns: (1) cluster name; (2) and (3) cluster right ascension and declination, respectively, in J2000 coordinates, taken from the X-ray catalogs (see Section 2); (4) cluster redshift; (5) $0.1-2.4 \mathrm{keV}$ band cluster X-ray luminosity within $R_{500}$; (6) $0.1-2.4 \mathrm{keV}$ band cluster X-ray luminosity within $R_{500}$ corrected for the contribution of the cool-core; (7) the ratio between the X-ray luminosity within the core and the total luminosity within $R_{500}$, cool-core clusters are indicated with ${ }^{c c}$; (8) $k$-corrected radio halo power at $1.4 \mathrm{GHz}$; (9) X-ray exposure in ks, with $\mathrm{P}=$ ROSAT PSPC, $\mathrm{H}=$ ROSAT HRI, and C $=$ Chandra ACIS-I; (10) the symbol $\sqrt{ }$ indicates the clusters present in the 15.5 month Planck catalog. References for the radio halo powers are ${ }^{v}$ Venturi et al. (2008); ${ }^{k}$ Kale et al. (2013); ${ }^{q}$ Guglielmino 2008, private communication; ${ }^{r}$ Russell et al. (2011); ${ }^{a}$ S. Giacintucci et al. (in preparation); ${ }^{b}$ Giacintucci et al. (2009); ${ }^{c}$ Kim et al. (1990); ${ }^{d}$ Clarke \& Ensslin (2006); ${ }^{e}$ Liang et al. (2000); ${ }^{f}$ Govoni et al. (2005); ${ }^{g}$ Farnsworth et al. (2013); ${ }^{h}$ Bonafede et al. (2009); van Weeren et al. (2009); ${ }^{i}$ Dallacasa et al. (2009); ${ }^{l}$ Macario et al. (2010); ${ }^{m}$ Bonafede et al. (2012); ${ }^{n}$ Giacintucci et al. (2013); ${ }^{o}$ Macario et al. (2011); ${ }^{p}$ Venturi et al. (2013). ${ }^{*}$ the X-ray luminosity of the Coma cluster is taken from O'Hara et al. (2006); ${ }^{* *}$ the X-ray luminosity of PLCKG171.9-40.7 is taken from Planck Collaboration et al. (2011c). 
1. $8 \mathrm{RH}$ from the EGRHS;

2. 29 clusters with radio upper limits from the EGRHS;

3. $6 \mathrm{RH}$ with ultra-steep spectra ( $\alpha>1.5$; USSRH hereafter); not to be compared to the upper limits (which were scaled at $1.4 \mathrm{GHz}$ with $\alpha=1.3$ );

4. 2 RH from the literature, A1995 and the Bullet cluster, which are in the same redshift and X-ray luminosity range of the EGRHS;

5. $9 \mathrm{RH}$ from the literature, which do not fulfill the EGRHS selection criteria (in redshift and X-ray luminosity).

The inclusion of $\mathrm{RH}$ from the literature is useful to have a sufficient leverage in radio/X-ray luminosities that may help to derive the scaling relations; however, the comparison between halos and upper limits will be performed only for clusters of EGRHS, which are in the same redshift range.

\section{DATA ANALYSIS}

In this section, we briefly describe the procedures undertaken to derive the radio and X-ray luminosities of clusters, to identify cool core clusters, and to analyze the cluster dynamical status. We also report measurements of the SZ signal found in the literature.

\subsection{Radio Power of Halos}

Table 1 reports the radio halo powers and upper limits for the clusters in the sample. We refer to the literature information (see notes to the table) for details and for the radio images.

For $12 \mathrm{RH}$ clusters in the sample we re-analyzed archival 1.4 GHz VLA-C and VLA-D array data (S. Giacintucci et al., in preparation). Another six clusters with giant RHs are published by our group and the data analysis was carried out following procedures similar to those described in this section. For the remaining seven RHs, the radio flux densities were taken from the literature. For those clusters we analyzed $(12+6)$, the flux density of the radio halo was measured from low angular resolution images obtained after subtraction of the contribution of the individual radio sources embedded in the diffuse emission. In particular, we identified the discrete radio galaxies in (or projected onto) and around the cluster region using the higher resolution images produced with the $\mathrm{C}$-array datasets (when available). The discrete radio sources were subtracted from the $u-v$ datasets, and the resulting $u-v$ visibilities were then used to image the radio halo emission at low resolution. In those cases where high-resolution data were not available to evaluate and subtract the contribution of individual sources, we produced images gapping the innermost region of the $u-v$ plane and used only the remaining long baselines $(\gtrsim 1-2 \lambda)$, which contain information on structures on angular scales smaller than the underlying large-scale radio halo. For extended sources, we used sets of images with different resolutions and/or $u-v$ ranges to determine their total extent and morphology. For each source and each cluster, we carefully checked that the total flux density subtracted from the $u-v$ data is consistent with the flux density measured on the images.

We measured the total flux density of the radio halos starting from the $3 \sigma$ contour level in the final images, then we progressively increased the extraction region until the integrated flux density reached a maximum value, and considered this maximum value as the total flux density of the halo. This procedure leads to an average increase of the halo flux density by only $\sim 5 \%$ with respect to the value within the $3 \sigma$ isocontour.
Finally, we calculated the corresponding radio power at the cluster redshift and applied a $k$-correction $(1+z)^{(1-\alpha)}$, where the spectral index is taken from the literature (references in Table 1), or was assumed to be $\alpha=1.3$ when not available. The errors on the diffuse radio flux density, $f_{H}$, account for the uncertainty in the calibration of the absolute flux density scale, the error due to the noise in the integration area, and the error due to the subtraction of the discrete radio sources in the halo region, as

$$
\sigma_{f_{H}}=\sqrt{\left(\delta_{\mathrm{cal}} f_{H}\right)^{2}+\left(\mathrm{rms} \sqrt{N_{\mathrm{beam}}}\right)^{2}+\sigma_{\mathrm{sub}}^{2}}
$$

where $\delta_{\text {cal }}$ is typically of the order of $5 \%-8 \%$, rms is the noise of the map, $N_{\text {beam }}$ is the number of independent beams in the halo region, and $\sigma_{\text {sub }}$ is the error due to the uncertainty in the source subtraction. The term $\sigma_{f_{H}}$ does not account for the uncertainty due to the missing short spacings in the $u-v$ coverage of the interferometric observations, and this may bias the flux densities toward lower values.

Upper limits to the diffuse radio flux of clusters without giant RH were reported in Venturi et al. (2008) and Kale et al. (2013) and scaled at $1.4 \mathrm{GHz}$ using a spectral index typical of RHs, $\alpha=1.3$.

\subsection{X-Ray Luminosities}

To derive the cluster X-ray luminosities we use ROSAT data, PSPC preferentially and HRI when PSPC data are not available. In those cases where ROSAT data are not available, we use Chandra data (see Table 1). We derive the X-ray luminosities inside $R_{500}$ centered on the centroid of the X-ray emission for all clusters. To estimate $R_{500}$ for our clusters, we searched the literature for information about the X-ray temperature and then applied the relations from Arnaud et al. (2005). We derived luminosities in the $0.1-2.4 \mathrm{keV}$ band in three different ways: (1) the entire cluster emission inside $R_{500}$, denoted as $L_{500}$; (2) the emission inside the aperture [0.15-1] $R_{500}$, denoted as $L_{500, \text { nc }}$; (3) $L_{500, \text { cor }}$, the X-ray luminosity inside $R_{500}$ corrected for the excess emission within $0.15 R_{500}$, due to the presence of a cooling core. For each cluster, $L_{500 \text {,cor }}$ is computed by performing a fit with a $\beta$-model to the cluster emission outside $0.15 R_{500}$, fixing $r_{c}=0.15 R_{500}$ (assuming that $r_{c} \simeq r_{\text {cool }} \simeq 0.15 R_{500}$, which is $\sim 100-200 \mathrm{kpc}$ for our sample) and evaluating the contribution of the model inside $0.15 R_{500}$. When the model fit underestimates the counts in the core, we correct the central region by using the fit to the X-ray brightness distribution outside $0.15 R_{500}$. We masked the detected point sources after a careful inspection of the cluster Chandra images (only in four cases we used ROSAT PSPC/HRI data). Following Russell et al. (2013), bright central point sources were identified and masked using the Chandra 5-8 keV images. The flux in the masked regions has been replaced by estimates based on the cluster best-fit model for the spatial brightness distribution. The obtained values of $L_{500}$ and $L_{500 \text {,cor }}$ are reported in Table 1.

\subsection{Identification of Cool-core Clusters}

In this section, we identify cool-core clusters in our sample to investigate possible biases that can be induced by cool-core clusters on scaling relations and bimodality.

As first measurement, we consider the X-ray surface brightness concentration parameter, defined as the ratio between the X-ray luminosity within the core region ( $L_{\text {core }}$, within $\left.0.15 R_{500}\right)$ and $L_{500}$ (e.g., Santos et al. 2008; Cassano et al. 2010, with slightly different definitions). In the literature, the concentration 

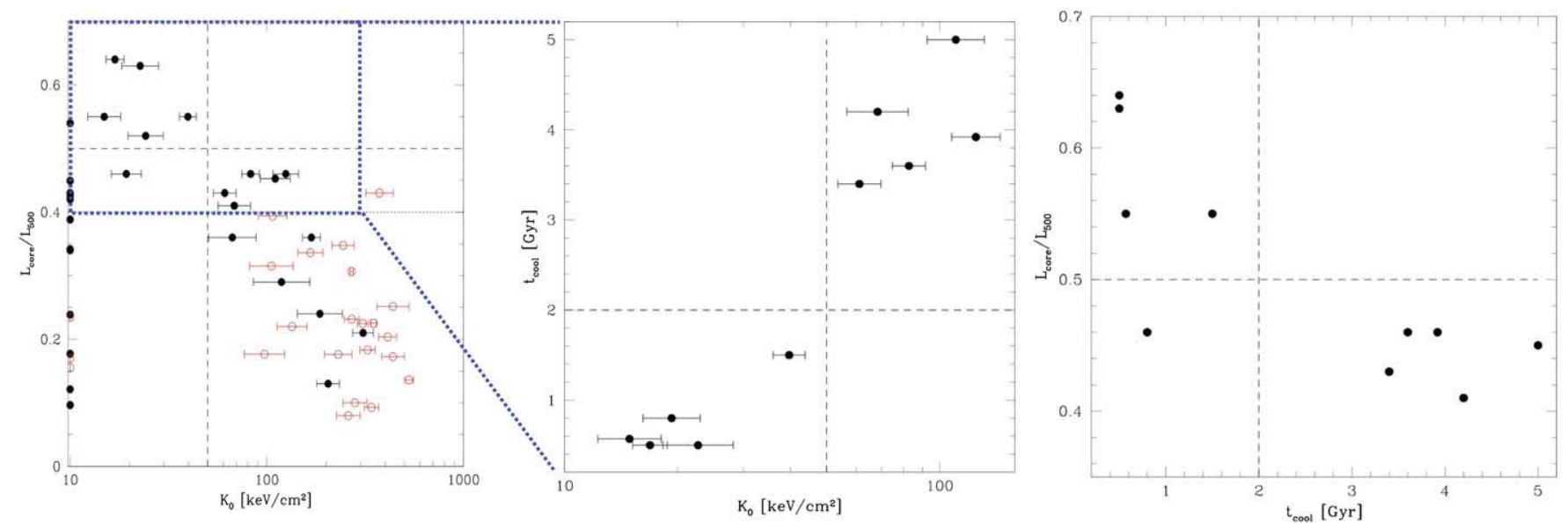

Figure 1. Left panel: $L_{\text {core }} / L_{500}$ versus $K_{0}$ for all clusters in our sample, for those clusters for which we do not find values of $K_{0}$ in Cavagnolo et al. (2009), we set $K_{0}=10 \mathrm{keV} \mathrm{cm}^{-2}$ (at the boundary of the plot). Clusters without giant RHs and clusters with giant RHs are reported as the black and red points, respectively. Central panel: $t_{\text {cool }}$ versus $K_{0}$ for all clusters with $L_{\text {core }} / L_{500}>0.4$ and $K_{0}<130 \mathrm{keV} \mathrm{cm}^{-2}$ (blue dashed region in the left panel); Right panel: $L_{\text {core }} / L_{500}$ versus $t_{\text {cool }}$ for all clusters with $L_{\text {core }} / L_{500}>0.4$ and $K_{0}<130 \mathrm{keV} \mathrm{cm}^{-2}$.

(A color version of this figure is available in the online journal.)

parameter has been used for a first identification of cool-core clusters in those cases where a spatially resolved spectroscopic analysis was not possible (e.g., in the case of high-redshift clusters; Santos et al. 2008) and to discriminate between merging clusters and more relaxed clusters (e.g., Cassano et al. 2010). A large value of this parameter indicates a large probability that the object has a cool core.

The derived values of the ratio $L_{\text {core }} / L_{500}$ are reported in Table 1 . Here we use the concentration parameter in combination with the central entropy $\left(K_{0}\right.$ in $\left.\mathrm{keV} \mathrm{cm}{ }^{-2}\right)$ and the central cooling time $\left(t_{\text {cool }}\right)$ to identify clusters with a cool-core. Values of $K_{0}<50 \mathrm{keV} \mathrm{cm}^{-2}$ (dashed vertical line in Figure 1) are used to identify cool-core clusters (e.g., Cavagnolo et al. 2009; Rossetti et al. 2011).

We inspected the sample of Cavagnolo et al. (2009) to find information about the central entropy for our clusters, and in Figure 1 (left panel), we report the distribution of clusters in the $L_{\text {core }} / L_{500}$ versus $K_{0}$ diagram; 13 of our clusters are not available in the Cavagnolo et al. sample, and in Figure 1 (left panel), they are reported with a value of $K_{0}=10 \mathrm{keV} \mathrm{cm}^{-2}$. We find the following.

1. Clusters with giant RHs (open red dots) have $L_{\text {core }} / L_{500}<$ $0.4^{14}$ and $K_{0}>90 \mathrm{keV} \mathrm{cm}^{-2}$.

2. Five clusters have $K_{0}<50 \mathrm{keV} \mathrm{cm}^{-2}$ and $L_{\text {core }} / L_{500}>$ 0.5: RXJ1532.9+3021, RXCJ1115.8+0129, Z2089, RXJ0439.0+0520, Z2701, and one, A2667, has $L_{\text {core }} / L_{500}=0.46$.

3. Five clusters have $50 \mathrm{keV} \mathrm{cm}^{-2}<K_{0}<130 \mathrm{keV} \mathrm{cm}^{-2}$ and $L_{\text {core }} / L_{500}>0.4: \mathrm{A} 611, \mathrm{~A} 2261, \mathrm{~A} 3088, \mathrm{~A} 1423$, and A2537.

As expected, clusters with giant RHs can be easily identified with merging clusters. To better understand whether the 11 clusters with $L_{\text {core }} / L_{500}>0.4$ and $K_{0}<130 \mathrm{keV} \mathrm{cm}^{-2}$ are cool-core or non cool-core clusters, we searched for information in the literature about their central cooling time $\left(t_{\text {cool }}\right)$ (Figure 1, central panel). ${ }^{15}$ Clusters with $K_{0}<50 \mathrm{keV} \mathrm{cm}^{-2}$ have $t_{\text {cool }}<$ 2 Gyr, while the others have $t_{\text {cool }}>3$ Gyr. Figure 1, right

\footnotetext{
14 The only exception is A1995 with $L_{\text {core }} / L_{500}=0.43$ (see also discussion in Section 6.2).

15 We do not find information about $t_{\text {cool }}$ in the literature for Z2089.
}

panel, also shows that clusters with $K_{0}<50 \mathrm{keV} \mathrm{cm}^{-2}$ and $t_{\text {cool }}<2$ Gyr all have $L_{\text {core }} / L_{500}>0.5$ (with the exception of A2667). Therefore, based on the combination of the three indicators, we identify cool-core clusters in our sample as those with $L_{\text {core }} / L_{500}>0.5$, i.e., clusters that emit more than $50 \%$ of their $L_{500}$ within their cores. ${ }^{16}$ Thus, we can conclude that there are seven cool-core clusters ${ }^{17}$ in our sample (these are marked with a ${ }^{c c}$ symbol in Column 7 of Table 1).

\subsection{Cluster Sunyaev-Zel'dovich Measurements}

Observations of clusters through their SZ-effect offer a valid alternative to $\mathrm{X}$-rays for the measure of the cluster mass, since the magnitude of the SZ effect is proportional to the integral along the line of sight of the cluster pressure, and hence is proportional to the cluster mass. The total SZ signal can be defined as

$$
\begin{aligned}
Y_{\Delta_{c}}= & D_{A}^{2} Y_{\mathrm{SZ}}=\left(\frac{\sigma_{T}}{m_{e} c^{2}}\right) \int_{R \leqslant R_{\Delta_{c}}} P d V \propto \\
& \times M_{\mathrm{gas}} T_{e}=f_{\mathrm{gas}} M_{\mathrm{tot}} T_{e},
\end{aligned}
$$

where $D_{A}$ is the angular diameter distance to the system, $\sigma_{T}$ is the Thomson cross-section, $c$ is the light speed, $m_{e}$ is the electron rest mass, $P=n_{e} k T_{e}$ is the electron pressure, $f_{\mathrm{gas}}$ is the gas mass fraction, and $M_{\text {tot }}$ is the total cluster mass. The integral in Equation (2) is performed over a sphere of radius, $R_{\Delta_{c}}$, which is the radius corresponding to a density contrast, $\Delta_{c} \rho_{c}(z)$. When the integration is performed over a sphere of radius $R_{500}$, the $\mathrm{SZ}$ signal is denoted with $Y_{500}$, which in the following paper will have the unit dimension of $\mathrm{Mpc}^{2}$.

For all clusters in Table 1, we search for information about the SZ signal in the recent all-sky PSZ cluster catalog, which contains all validated clusters from the first 15.5 months of Planck satellite observations (Planck Collaboration et al. 2013b). Considering only clusters belonging to the EGRHS subsample, we find that 11/11 RH clusters and 19 out of 29 clusters with upper

\footnotetext{
16 We also consider A2667 as a cool-core cluster, since it has an estimated central entropy of $K_{0} \approx 19 \mathrm{keV} \mathrm{cm}^{-2}$ and a central cooling time $t_{\text {cool }} \approx 1 \mathrm{Gyr}$ and it is classified as a cool core cluster by Zhang et al. (2007).

17 The cluster Z348 has no information about $K_{0}$ in the literature, but since it has $L_{\text {core }} / L_{500}=0.54$, we can identify it as a cool-core cluster.
} 
limits are contained in the PSZ catalog. Among the 10 clusters not present in the PSZ catalog, 5 are cool-core clusters; therefore, only 2 out of 7 cool-core clusters of our sample are detected by Planck. The remaining $14 \mathrm{RH}$ clusters from the literature are also contained in PSZ catalog.

We obtain a subsample of 44 clusters (25 halos and 19 upper limits) for which Planck measurements of $Y_{500}$ are available (see Table 2).

For the same clusters, we also find information in the PSZ catalog about the values of $M_{500}$. These are obtained from $Y_{500}$ as described in Planck Collaboration et al. (2013b, Section 7.2.2) and are reported in Table 2.

\subsection{Cluster Dynamical Status}

For clusters belonging to the EGRHS with information about $Y_{500}$ and $M_{500}$ (see Table 2) we make use of Chandra archival data to determine the cluster dynamical status. ${ }^{18}$ We produce $\mathrm{X}$-ray images in a standard manner using CIAO 4.3 (with calibration files from CALDB 4.4.1) in the $0.5-2 \mathrm{keV}$ band. We adopted an algorithm for an automatic detection of the point sources, which are then removed from the images. Following Cassano et al. (2010), we study the cluster substructure on the $\mathrm{RH}$ scale analyzing the surface brightness inside an aperture radius of $500 \mathrm{kpc}$, since we are interested in the cluster dynamical properties on the scales where the energy is most likely dissipated. We use two methods: the emission centroid shift (e.g., Mohr et al. 1993; Poole et al. 2006; O'Hara et al. 2006; Ventimiglia et al. 2008; Maughan et al. 2008; Böhringer et al. 2010) and the surface brightness concentration parameter (e.g., Santos et al. 2008).

The centroid shift, $w$, is computed in a series of circular apertures centered on the cluster X-ray peak and is defined as the standard deviation of the projected separation between the peak and the centroid in units of $R_{\text {ap }}$ as (Poole et al. 2006; Maughan et al. 2008)

$$
w=\left[\frac{1}{N-1} \sum\left(\Delta_{i}-\langle\Delta\rangle\right)^{2}\right]^{1 / 2} \times \frac{1}{R_{\mathrm{ap}}},
$$

where $\Delta_{i}$ is the distance between the X-ray peak and the centroid of the $i$ th aperture.

Following Santos et al. (2008), the concentration parameter, $c$, is defined as the ratio of the peak over the ambient surface brightness, $S$, as

$$
c=\frac{S(r<100 \mathrm{kpc})}{S(<500 \mathrm{kpc})} \text {. }
$$

We use the concentration parameter to differentiate galaxy clusters with a compact core (i.e., core not disrupted from a recent merger event) from clusters with a spread distribution of gas in the core (i.e., core disturbed from a recent merger episode).

Cassano et al. (2010) showed that, considering the median value of each parameter, $w=0.012$ and $c=0.2$, it was possible to separate the sample between RH merging clusters $(w>0.012$ and $c<0.2)$ and more relaxed clusters without RHs $(w<0.012$ and $c>0.2)$. We will use these values as reference for our sample.

\footnotetext{
18 With the exception of A2697 for which Chandra data are not available.
}

Table 2

\begin{tabular}{|c|c|c|c|}
\hline Cluster Name & Index & $\begin{array}{c}\log \left(Y_{500}\right) \\
\left(\mathrm{Mpc}^{2}\right)\end{array}$ & $\begin{array}{c}\log \left(M_{500}\right) \\
\left(M_{\odot}\right) \\
\end{array}$ \\
\hline \multicolumn{4}{|l|}{ Upper limits (EGRHS) } \\
\hline A2697 & 315 & $-4.150 \pm 0.077$ & $14.78 \pm 0.04$ \\
\hline A141 & 599 & $-4.379 \pm 0.120$ & $14.65 \pm 0.07$ \\
\hline A3088 & 744 & $-4.062 \pm 0.065$ & $14.83 \pm 0.04$ \\
\hline RXCJ1115.8 + 0129 & 881 & $-4.087 \pm 0.087$ & $14.80 \pm 0.05$ \\
\hline A2631 & 297 & $-4.029 \pm 0.067$ & $14.84 \pm 0.04$ \\
\hline A2645 & 254 & $-4.288 \pm 0.099$ & $14.70 \pm 0.06$ \\
\hline A2667 & 94 & $-4.054 \pm 0.055$ & $14.83 \pm 0.03$ \\
\hline RXJ0142.0 + 2131 & 500 & $-4.134 \pm 0.102$ & $14.78 \pm 0.06$ \\
\hline A267 & 541 & $-4.301 \pm 0.108$ & $14.69 \pm 0.06$ \\
\hline RXJ0439.0+0715 & 640 & $-4.181 \pm 0.096$ & $14.76 \pm 0.05$ \\
\hline A611 & 623 & $-4.162 \pm 0.081$ & $14.77 \pm 0.05$ \\
\hline A781 & 654 & $-4.097 \pm 0.072$ & $14.80 \pm 0.04$ \\
\hline A1423 & 610 & $-4.143 \pm 0.064$ & $14.78 \pm 0.04$ \\
\hline A 1576 & 460 & $-4.143 \pm 0.063$ & $14.78 \pm 0.04$ \\
\hline A2146 & 359 & $-4.495 \pm 0.080$ & $14.58 \pm 0.05$ \\
\hline A2261 & 174 & $-3.991 \pm 0.048$ & $14.87 \pm 0.03$ \\
\hline RXJ2228.6 + 2037 & 275 & $-3.917 \pm 0.072$ & $14.89 \pm 0.04$ \\
\hline A2537 & 247 & $-4.120 \pm 0.080$ & $14.79 \pm 0.04$ \\
\hline S780 & 1185 & $-3.957 \pm 0.062$ & $14.89 \pm 0.03$ \\
\hline S780 & 1185 & $-3.957 \pm 0.062$ & $14.89 \pm 0.03$ \\
\hline \multicolumn{4}{|l|}{ Radio Halos (EGRHS) } \\
\hline A2744 & 26 & $-3.778 \pm 0.041$ & $14.98 \pm 0.02$ \\
\hline A0209 & 558 & $-3.916 \pm 0.041$ & $14.91 \pm 0.02$ \\
\hline A2163 & 19 & $-3.374 \pm 0.019$ & $15.22 \pm 0.01$ \\
\hline RXCJ2003.5-2323 & 46 & $-3.967 \pm 0.068$ & $14.87 \pm 0.04$ \\
\hline A520 & 655 & $-4.030 \pm 0.062$ & $14.85 \pm 0.04$ \\
\hline A773 & 578 & $-4.026 \pm 0.049$ & $14.85 \pm 0.03$ \\
\hline A1758a & 389 & $-3.922 \pm 0.044$ & $14.90 \pm 0.03$ \\
\hline A2219 & 242 & $-3.681 \pm 0.026$ & $15.04 \pm 0.01$ \\
\hline $\mathrm{A} 521^{U}$ & 688 & $-4.040 \pm 0.070$ & $14.83 \pm 0.04$ \\
\hline $\mathrm{A} 697^{U}$ & 628 & $-3.640 \pm 0.032$ & $15.06 \pm 0.02$ \\
\hline $\mathrm{A} 1300^{U}$ & 960 & $-3.839 \pm 0.053$ & $14.95 \pm 0.03$ \\
\hline \multicolumn{4}{|l|}{ Radio Halos (literature) } \\
\hline CL0016 + 1609 & 408 & $-3.813 \pm 0.077$ & $14.94 \pm 0.04$ \\
\hline A1914 & 224 & $-4.045 \pm 0.039$ & $14.84 \pm 0.02$ \\
\hline A665 & 533 & $-3.914 \pm 0.037$ & $14.92 \pm 0.02$ \\
\hline A545 & 707 & $-4.397 \pm 0.112$ & $14.64 \pm 0.06$ \\
\hline Coma & 187 & $-4.281 \pm 0.030$ & $14.72 \pm 0.02$ \\
\hline A2256 & 407 & $-4.135 \pm 0.022$ & $14.80 \pm 0.01$ \\
\hline A2255 & 325 & $-4.288 \pm 0.028$ & $14.71 \pm 0.02$ \\
\hline A2319 & 252 & $-3.900 \pm 0.020$ & $14.93 \pm 0.01$ \\
\hline MCSJ0717.5 + 3745 & 608 & $-3.612 \pm 0.049$ & $15.05 \pm 0.03$ \\
\hline Bullet & 920 & $-3.577 \pm 0.025$ & $15.09 \pm 0.02$ \\
\hline A1995 & 337 & $-4.257 \pm 0.075$ & $14.71 \pm 0.04$ \\
\hline MCSJ1149.5 + 2223 & 765 & $-3.824 \pm 0.072$ & $14.93 \pm 0.04$ \\
\hline PLCK G171.9-40.7 ${ }^{U}$ & 591 & $-3.666 \pm 0.039$ & $15.05 \pm 0.02$ \\
\hline $\mathrm{A} 754^{U}$ & 801 & $-4.095 \pm 0.023$ & $14.82 \pm 0.01$ \\
\hline
\end{tabular}

Observed Cluster SZ Properties

Notes. Columns: (1) radio properties; (2) cluster name; (3) index indicating the position in the Planck validation catalog; (4) logarithmic value of $Y_{500}$ in $\mathrm{Mpc}^{2}$, with $68 \%$ errors; (5) logarithmic value of $M_{500}$ in solar masses, with $68 \%$ errors. Clusters marked with ${ }^{U}$ are those hosting USSRHs. All the $M_{500}$ and $Y_{500}$ values refer to Planck Collaboration et al. (2013b; from the Web site: http://szcluster-db.ias.u-psud.fr); with the exception of the cluster PLCK G171.9-40.7, whose values are taken from Planck Collaboration et al. (2011c).

\subsection{Fitting Procedure}

Here we describe the procedure used in the next sections to investigate the presence of scaling relations between independent measurements, i.e., the RH power and the cluster thermal quantities $\left(L_{500}, L_{500, \text { cor }}, Y_{500}\right.$, and $\left.M_{500}\right)$. For each set of 
observables, we fit a power-law relation using linear regression in the log-log space by adopting the BCES-bisector and the BCES-orthogonal regression algorithms (Akritas \& Bershady 1996) which treat the variables symmetrically and take into account measurement errors in both variables and intrinsic scatter in the data. Performing Monte Carlo simulations to test the performances of different regression methods, Isobe et al. (1990) recommended the use of BCES-bisector in the case one would like to treat the variables symmetrically. Consequently, we will consider the BCES-bisector as the reference method.

Since we also have upper limits on $P_{1.4}$, in those cases where upper limits and detections are not clearly separated, we also use a regression analysis based on the parametric EM (ExpectationMaximization) algorithm that is implemented in the ASURV package (Isobe et al. 1986) and deals with "censored data," upper limits.

Assuming a linear relation of the form $Y=a X+b$, and a sample of $N$ data points $\left(Y_{i}, X_{i}\right)$ with errors $\sigma_{Y i}$ and $\sigma_{X i}$, we estimate the raw scatter using the error weighted orthogonal distances to the regression line (e.g., Pratt et al. 2009; Biffi et al. 2013):

$$
\sigma_{\text {raw }}^{2}=\frac{1}{N-2} \sum_{i=1}^{N} w_{i}\left(Y_{i}-a X_{i}-b\right)^{2},
$$

where

$$
w_{i}=\frac{1 / \sigma_{i}^{2}}{(1 / N) \sum_{i=1}^{N} 1 / \sigma_{i}^{2}} \quad \text { and } \quad \sigma_{i}^{2}=\sigma_{Y_{i}}^{2}+a^{2} \sigma_{X_{i}}^{2} .
$$

Since we are dealing with a limited sample, the regression line obtained for our data is a sample regression line that can deviate from the (unknown) true regression line. To evaluate the variation of our best-fit relation about the true regression line, we estimate the $95 \%$ confidence interval for the mean value of $\langle Y\rangle$ at a given $X$, i.e., the area that has a $95 \%$ chance of containing the true regression line. For a given value of the $X$ variable the 95\% confidence region around the mean $\langle Y\rangle$ (which is given by the best-fit relation: $\langle Y\rangle=a X+b)$ is $\langle Y\rangle \pm \Delta Y$, where

$$
\Delta Y= \pm 1.96 \sqrt{\sum_{i=1}^{N} \frac{\left(Y_{i}-Y_{m}\right)^{2}}{N-2}} \sqrt{\left(\frac{1}{N}+\frac{\left(X-X_{m}\right)^{2}}{\sum_{i=1}^{N}\left(X_{i}-X_{m}\right)^{2}}\right)}
$$

where for each observed $X_{i}, Y_{m}=a X_{i}+b$, and $X_{m}=$ $\sum_{i=1}^{N} X_{i} / N$.

\section{EXPECTED SCALING RELATIONS}

Scaling relations between the synchrotron radio power of halos and the cluster thermal properties (mass, X-ray luminosity, temperature) are expected in theoretical models for the formation of giant RHs. In this section, we briefly summarize the basic theoretical expectations for the scalings.

\subsection{Secondary Models}

In the simplest scenario for the formation of giant RHs in clusters, the electrons responsible for the synchrotron emission are secondary products of the hadronic interaction between thermal and cosmic ray protons. In this model, following the formalism by Kushnir et al. (2009), the scaling between the synchrotron radio power and the cluster [0.1-2.4] keV X-ray luminosity is expected to be $v P_{v}^{\text {syn }} \propto L_{X}^{\frac{\alpha_{L}+0.5}{\alpha_{L}-0.6}}$, where $\alpha_{L}$ is the slope of the $L-T$ relation. For $\alpha_{L} \simeq 2.5-3$ (e.g., Markevitch 1998; Arnaud \& Evrard 1999; Reiprich \& Böhringer 2002; Pratt et al. 2009), one obtains

$$
v P_{v}^{\mathrm{syn}} \propto L_{X}^{1.58-1.46} .
$$

This is valid under the assumption that the relevant radiation losses for the secondary electrons are synchrotron losses, i.e., assuming that the average magnetic field strength in the halo volume is $B>B_{\mathrm{CMB}} \simeq 3.2(1+z)^{2} \mu \mathrm{G}$. Lower magnetic field values are disfavored by the combination of Planck and Fermi data with radio observations (e.g., Jeltema \& Profumo 2011; Planck Collaboration et al. 2013a; Brunetti et al. 2012).

Since $Y_{500}$ is found to scale as $Y_{500} \propto L_{X}^{1.02 \pm 0.07}$ (e.g., Planck Collaboration et al. 2011b), this model predicts

$$
v P_{v}^{\text {syn }} \propto\left(Y_{500}\right)^{1.55-1.43} \text {. }
$$

\subsection{Turbulent Re-acceleration Models}

In the case of the turbulent re-acceleration scenario, the derivation of scaling relations is less straightforward, due to our poor knowledge of the details of the microphysics of the ICM. A simple approach to derive scaling relations in this model is presented in Cassano et al. (2007). Under quasistationary conditions, the energy flux of the turbulence which goes into relativistic electrons is reradiated via synchrotron and IC mechanisms. The injection rate of the turbulence generated during a merger in the RH volume can be estimated as $\dot{\varepsilon_{t}} \propto$ $\rho_{H} \times v^{2} / \tau_{\text {cros }}$, where $\rho_{H}$ is the ICM mean density in the RH volume, $v$ is the cluster-cluster impact velocity, and $\tau_{\text {cros }}$ is the cluster crossing time. As in the case of secondary models, it is assumed that the ratio between the energy densities in relativistic particles and thermal plasma does not change in any systematic way with cluster mass (or temperature) among RH clusters. Under this hypothesis, the synchrotron radio power is $v P_{v}^{\text {syn }} \propto\left(M_{H} \sigma_{H}^{3}\right) / \mathcal{F}\left(z, M_{H}, b_{H}\right)$, where $\mathcal{F}\left(z, M_{H}, b_{H}\right)=$ $\left[1+\left(3.2(1+z)^{2} / B_{H}\right)^{2}\right]$, and $M_{H}, \sigma_{H}$, and $B_{H}$ are the total cluster mass, the cluster velocity dispersion, and the average magnetic field strength within the RH size $\left(R_{H}\right)$, respectively (Cassano et al. 2007). The expression $\mathcal{F}$ is constant in the asymptotic limit $B_{H}^{2} \gg B_{\mathrm{cmb}}^{2}$, or when the magnetic field in the $\mathrm{RH}$ region is independent of the cluster mass. In this case, $v P_{v}^{\text {syn }} \propto M_{H}^{1.8}$. Assuming the scalings $M_{H} \propto R_{H}^{2.17}$ (Cassano et al. 2007) and $R_{H} \propto R_{500}^{3.1}$ (Basu 2012), one has

$$
v P_{v}^{\text {syn }} \propto M_{500}^{4.0}
$$

and considering the scaling $M_{500} \propto Y_{500}^{1 / 1.74}$, one has

$$
v P_{v}^{\text {syn }} \propto Y_{500}^{2.3}
$$

which is steeper than that predicted by "secondary models." Reacceleration modes also allow the case $B_{H}^{2} \ll B_{\text {cmb }}^{2}$, without tension with $\gamma$-ray upper limits (e.g., Brunetti et al. 2012), and in this case one has $\mathcal{F}^{-1} \propto M_{H}^{2 b_{H}}$, which implies a correlation even steeper than that obtained in the previous case.

Besides the details of the slopes of the thermal-non-thermal scaling relations expected from a different origin of the emitting electrons, an important difference between the two scenarios is the expected dispersion of the correlations. Re-acceleration models predict a variety of spectral shapes of RHs, including very steep spectra (e.g., Cassano et al. 2006; Brunetti et al. 2008), which imply a substantial dispersion in the correlations (Kushnir et al. 2009; Brunetti et al. 2009) and an increase of the scatter at low observing frequency (Cassano 2010). 

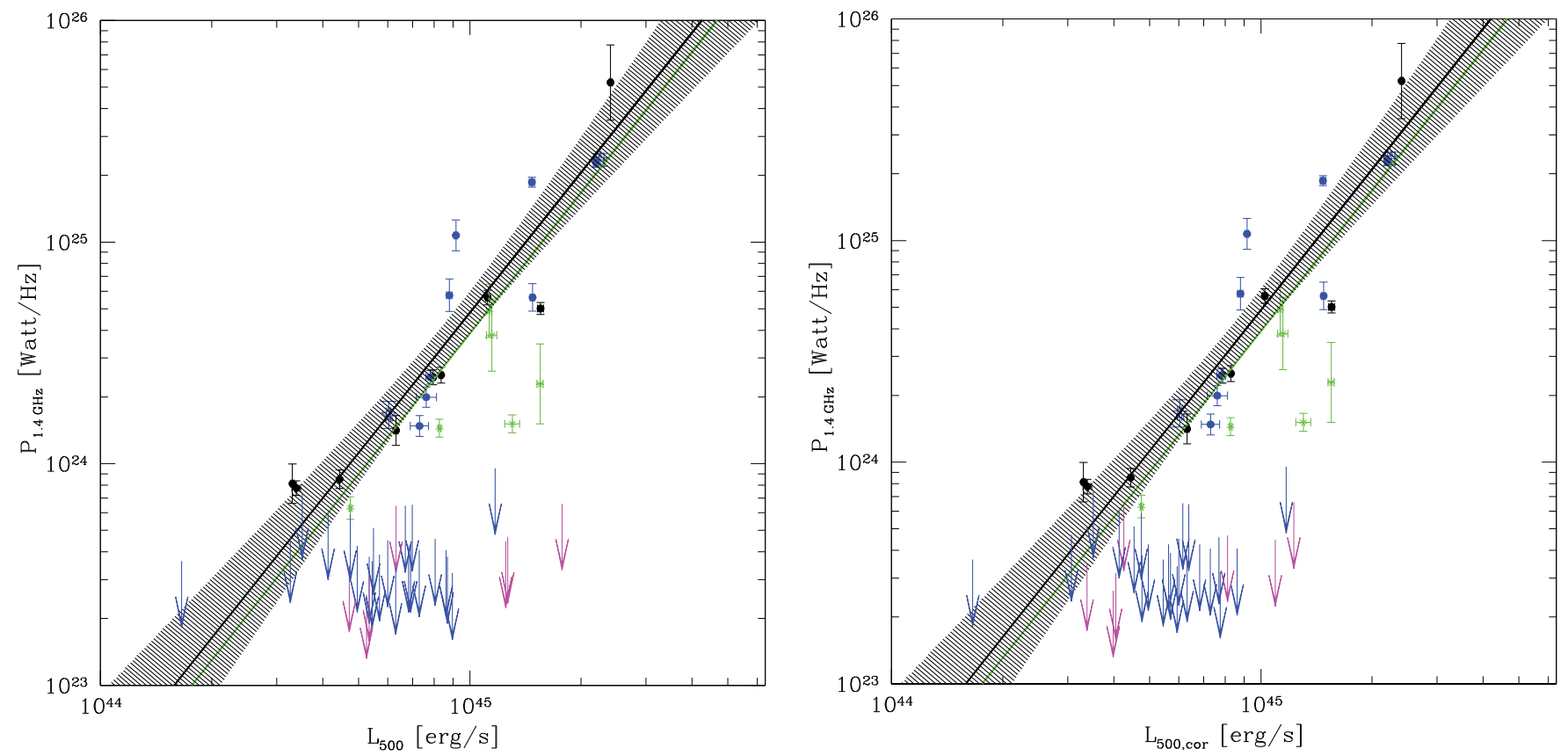

Figure 2. Left panel: distribution of clusters in the $P_{1.4}-L_{500}$ plane. Right panel: distribution of clusters in $P_{1.4}-L_{500 \text {,cor }}$ plane. In both panels, different symbols indicate halos belonging to the EGRHS (blue filled dots); halos from the literature (black open dots); halos with very steep spectra (USSRH, green asterisks); A1995 and Bullet cluster (blue stars); cool core clusters belonging to the EGRHS (magenta arrows). Best-fit relations to giant RHs only (black lines) and to all RHs (including USSRH, green dashed lines) are reported. The 95\% confidence regions of the best-fit relations obtained for giant RHs only are also reported (shadowed regions).

(A color version of this figure is available in the online journal.)

\section{RADIO-X-RAY LUMINOSITY CORRELATION AND THE BIMODALITY}

It is well known that the radio luminosity of halos at $1.4 \mathrm{GHz}$ scales with the X-ray luminosity of the hosting clusters (e.g., Liang et al. 2000; Feretti 2002, 2003; Enßlin \& Röttgering 2002; Cassano et al. 2006; Brunetti et al. 2009; Giovannini et al. 2009). This correlation has been used to claim that a correlation should also exist between the radio power and the virial mass of the host cluster (e.g., Cassano et al. 2006). Deep upper limits to the radio flux density of clusters with no $\mathrm{RH}$ emission at $610 \mathrm{MHz}$, which were a factor of $\sim 3-20$ below the correlation, were obtained from the GRHS and its extension ${ }^{19}$ allowing to validate the correlation itself and to discover the radio bimodality (e.g., Brunetti et al. 2007).

In previous papers, the distribution of galaxy clusters in the radio-X-ray luminosity diagram, and the scaling relation between the two quantities, were based on non-homogeneous radio and X-ray measurements. In particular, the radio luminosities of halos were collected from the literature and X-ray luminosities were taken from RASS-based cluster catalogs. Here we recomputed the radio flux densities of well known RHs by reanalyzing observations from the archives (as outlined in Section 3.1). For all clusters, we computed the 0.1-2.4 keV X-ray luminosities within $R_{500}$ from pointed ROSAT and Chandra observations (see Section 3.2).

In Figure 2, (left panel) we show the distribution of clusters in the $P_{1.4}-L_{500}$ diagram. We report with different colors clusters belonging to the EGRHS (blue points and blue and magenta arrows) and halos from the literature (black points). This is necessary, since the comparison between RH powers and upper limits makes sense only for those clusters observed

19 Previous attempts to compare upper limits and the correlation can be found in Dolag (2006). within the same redshift range, and this is possible only for clusters belonging to the EGRHS. Halos from the literature follow the same distribution of halos from the EGRHS, and thus we use them to draw the correlation. RH clusters appear to follow a well-defined correlation between the halo radio power and $L_{500}$. Being steeper than other halos, ultra-steep spectrum RH (green asterisks) are, in general, under-luminous with respect to this correlation. Remember that the position of USSRH in the $P_{1.4}-L_{500}$ diagram cannot be compared with that of the upper limits as the latter were scaled at $1.4 \mathrm{GHz}$ using $\alpha=1.3$. We find a bimodal distribution of clusters with the presence of two distinct populations, that of radio-halo clusters and that of radio-quiet clusters. For values of $L_{500} \gtrsim 5 \times 10^{44} \mathrm{erg} \mathrm{s}^{-1}$, clusters with upper limits to the radio power (blue and magenta arrows) are all located below the $95 \%$ confidence region of the correlation.

As the EGRHS is based on X-ray-selected clusters, one may suspect that the bimodality could be caused by the presence of cool-core clusters, which are brighter in X-ray and do not host giant radio-halos. With the idea to test the bimodality against the presence of cool-core clusters in the EGRHS, we derive the distribution of clusters in the $P_{1.4}-L_{500 \text {, cor }}$ diagram (Figure 2, right panel). We highlight the position of cool-core clusters (identified as outlined in Section 3.3, magenta arrows in Figures 2). As expected, the X-ray luminosity of cool-core clusters is significantly reduced going from $L_{500}$ to $L_{500, \text { cor }}$.

However, the bimodal behavior in the halo radio power also

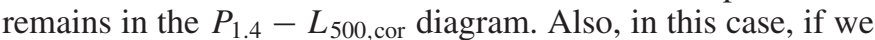
restrict to clusters with $L_{500 \text {,cor }} \gtrsim 5 \times 10^{44} \mathrm{erg} \mathrm{s}^{-1}$, upper limits are all below the $95 \%$ confidence region of the correlation. We may thus conclude that the observed radio bimodality is not driven by the presence of cool-core clusters without diffuse radio emission in the EGRHS. We fit the observed $P_{1.4}-L_{500}$ 
and $P_{1.4}-L_{500, \text { cor }}$ relation with a power-law of the generic form

$$
\log \left(\frac{P_{1.4}}{10^{24.5}{\text { Watt } \mathrm{Hz}^{-1}}^{2}}\right)=B \log \left(\frac{L_{X}}{10^{45} \mathrm{erg} \mathrm{s}^{-1}}\right)+A,
$$

where $L_{X}$ is $L_{500}$ or $L_{500, \text { cor }}$. The fit was performed using linear regression in the log-log space by adopting both the BCES-bisector and BCES-orthogonal methods (as discussed in Section 3.6). The results of the fit, together with that from 1000 bootstrap resamples, are reported in Table 3. The slope of the correlation is $\sim 2.1 \pm 0.2$ and $\sim 2.2 \pm 0.2$ in the BCES-bisector and BCES-orthogonal cases, respectively, consistent with that found in previous studies (e.g., Brunetti et al. 2009). The bestfit relation has a lower normalization and a larger $\sigma_{\text {raw }}$ when USSRH are included in the fit (see Table 3).

\section{RADIO-SZ SCALING RELATIONS}

As discussed in Section 3.4, observations of clusters through their SZ-effect may provide a powerful method to measure the cluster masses. Recently, Basu (2012) found a correlation between the radio power of clusters with RHs and the integrated Compton parameter derived from the Planck ESZ catalog (Planck Collaboration et al. 2011a) in the form $P_{1.4} \propto Y_{5 R_{500}}^{2}$, where $Y_{5 R_{500}}$ is the integral of the SZ signal within a radius of $5 R_{500} \cdot{ }^{20}$ Basu (2012) found indication for a weaker or lack of bimodality based on the fact that only four clusters from the GRHS with radio upper limits were found in the Planck ESZ catalog, while almost all RH of the GRHS have counterparts in the same catalog. Basu (2012) suggested that a possible reason for the lack of bimodality in SZ could be due to the fact that $\mathrm{X}$-ray selected cluster samples are biased towards the detection of X-ray luminous, but not necessarily massive, clusters, while the SZ tends to be more "mass-limited." In this picture, clusters with radio upper limits that are not detected by Planck should be less massive systems (with respect to those hosting giant RHs) with cool-cores. These clusters would appear brighter in X-ray because of the presence of a cool core, causing an apparent bimodality in the $P_{1.4}-L_{500}$ plane. However, as we have shown in Section 5, even when we consider the X-ray luminosity excising the cool core, we find a clear bimodality in the radio-X-ray plane (Figure 2, right panel).

The all-sky PSZ catalog that we are using in this paper, is six times the size of the Planck ESZ catalog (Planck Collaboration et al. 2013b) used by Basu (2012), and $~ 80 \%$ complete for $M_{500} \gtrsim 6 \times 10^{14} M_{\odot}$ at $z \simeq 0.2-0.35$, typical mass and redshift ranges of the EGRHS clusters. In Figure 3, we show the distribution of the 44 clusters of our sample belonging to the PSZ catalog (see Section 3.4), in the $P_{1.4}-M_{500}$ (left panel) and $P_{1.4}-Y_{500}$ (right panel) diagrams. We show with different colors clusters belonging to the EGRHS (blue points and blue and magenta arrows), halos from the literature (black points), and halos with ultra-steep radio spectra (green asterisks). The comparison between RHs and upper limits can be performed only for clusters belonging to the EGRHS, while the RHs from the literature are added to better determine the correlations. We find clear correlations between $P_{1.4}$ and $M_{500}$ and $Y_{500}$ parameters. Using the BCES regression method, we fit the observed $P_{1.4}-Y_{500}$ and $P_{1.4}-M_{500}$ relation with the following power laws:

$$
\log \left(\frac{P_{1.4}}{10^{24.5}{\text { Watt } \mathrm{Hz}^{-1}}}\right)=B \log \left(\frac{Y_{500}}{10^{-4} \mathrm{Mpc}^{2}}\right)+A
$$

\footnotetext{
${ }^{20} Y_{5 R_{500}}$ can be rescaled to $Y_{500}$ for the fiducial GNFW model as $Y_{5 R_{500}}=1.79 \times Y_{500}$ (Arnaud et al. 2010).
}

Table 3

\begin{tabular}{|c|c|c|c|c|c|c|c|}
\hline Method & $B$ & $\operatorname{err}(B)$ & $A$ & $\operatorname{err}(A)$ & $\sigma_{\text {raw }}$ & $r_{s}$ & $P$ \\
\hline \multicolumn{8}{|c|}{$P_{1.4}-L_{500}$} \\
\hline \multicolumn{8}{|l|}{ RH+USS } \\
\hline BCES bisector & 2.11 & 0.20 & 0.088 & 0.056 & 0.23 & 0.83 & $2.32 \times 10^{-7}$ \\
\hline Bootstrap & 2.11 & 0.21 & 0.083 & 0.058 & & & \\
\hline BCES orthogonal & 2.35 & 0.25 & 0.094 & 0.058 & & & \\
\hline Bootstrap & 2.37 & 0.31 & 0.089 & 0.062 & & & \\
\hline \multicolumn{8}{|l|}{ RH only } \\
\hline BCES bisector & 2.10 & 0.17 & 0.181 & 0.048 & 0.20 & 0.95 & $1.03 \times 10^{-7}$ \\
\hline Bootstrap & 2.11 & 0.19 & 0.176 & 0.049 & & & \\
\hline BCES orthogonal & 2.20 & 0.18 & 0.185 & 0.049 & & & \\
\hline Bootstrap & 2.21 & 0.23 & 0.180 & 0.049 & & & \\
\hline \multicolumn{8}{|c|}{$P_{1.4}-L_{500, \text { cor }}$} \\
\hline \multicolumn{8}{|l|}{$\mathrm{RH}+\mathrm{USS}$} \\
\hline BCES bisector & 2.11 & 0.20 & 0.091 & 0.056 & 0.23 & 0.83 & $2.32 \times 10^{-7}$ \\
\hline Bootstrap & 2.12 & 0.22 & 0.085 & 0.060 & & & \\
\hline BCES orthogonal & 2.35 & 0.25 & 0.098 & 0.058 & & & \\
\hline bootstrap & 2.38 & 0.31 & 0.094 & 0.065 & & & \\
\hline \multicolumn{8}{|l|}{ RH only } \\
\hline BCES-bisector & 2.11 & 0.16 & 0.186 & 0.048 & 0.20 & 0.95 & $1.03 \times 10^{-9}$ \\
\hline Bootstrap & 2.11 & 0.18 & 0.184 & 0.050 & & & \\
\hline BCES orthogonal & 2.20 & 0.18 & 0.190 & 0.049 & & & \\
\hline Bootstrap & 2.22 & 0.22 & 0.187 & 0.052 & & & \\
\hline \multicolumn{8}{|c|}{$P_{1.4}-M_{500}$} \\
\hline \multicolumn{8}{|l|}{ RH+USS } \\
\hline BCES bisector & 3.70 & 0.56 & 0.009 & 0.074 & 0.37 & 0.73 & $3.98 \times 10^{-5}$ \\
\hline Bootstrap & 3.73 & 0.64 & 0.011 & 0.079 & & & \\
\hline BCES orthogonal & 5.05 & 0.99 & 0.002 & 0.094 & & & \\
\hline Bootstrap & 5.27 & 1.33 & -0.002 & 0.107 & & & \\
\hline \multicolumn{8}{|l|}{ RH only } \\
\hline BCES-bisector & 3.77 & 0.57 & 0.125 & 0.076 & 0.35 & 0.81 & $2.50 \times 10^{-5}$ \\
\hline Bootstrap & 3.84 & 0.66 & 0.126 & 0.079 & & & \\
\hline BCES orthogonal & 4.51 & 0.78 & 0.129 & 0.087 & & & \\
\hline Bootstrap & 4.62 & 0.90 & 0.131 & 0.092 & & & \\
\hline \multicolumn{8}{|c|}{$P_{1.4}-Y_{500}$} \\
\hline \multicolumn{8}{|l|}{$\mathrm{RH}+\mathrm{USS}$} \\
\hline BCES bisector & 2.02 & 0.28 & -0.131 & 0.070 & 0.35 & 0.74 & $2.66 \times 10^{-5}$ \\
\hline Bootstrap & 2.03 & 0.30 & -0.133 & 0.069 & & & \\
\hline BCES orthogonal & 2.48 & 0.43 & -0.167 & 0.089 & & & \\
\hline Bootstrap & 2.55 & 0.51 & -0.177 & 0.100 & & & \\
\hline \multicolumn{8}{|l|}{ RH only } \\
\hline BCES-bisector & 2.05 & 0.28 & -0.014 & 0.068 & 0.32 & 0.83 & $1.26 \times 10^{-5}$ \\
\hline Bootstrap & 2.07 & 0.30 & -0.016 & 0.072 & & & \\
\hline BCES orthogonal & 2.28 & 0.35 & -0.027 & 0.073 & & & \\
\hline Bootstrap & 2.30 & 0.38 & -0.030 & 0.079 & & & \\
\hline \multicolumn{8}{|l|}{$\mathrm{RH}+\mathrm{UL}$} \\
\hline EM algorithm & 2.77 & 0.54 & -0.55 & 0.13 & & & \\
\hline \multicolumn{8}{|l|}{ RH only } \\
\hline EM algorithm & 1.70 & 0.26 & 0.006 & 0.068 & & & \\
\hline
\end{tabular}

Best-fit Parameters of Scaling Relations

Note. The last two columns gives the Spearman's rank correlation coefficient, $r_{s}$, and the related probability of no correlation.

and

$$
\log \left(\frac{P_{1.4}}{10^{24.5}{\text { Watt } \mathrm{Hz}^{-1}}}\right)=B \log \left(\frac{M_{500}}{10^{14.9} M_{\odot}}\right)+A .
$$

Results of the fits, together with those from 1000 bootstrap resamples, are reported in Table 3 . The slope of the $P_{1.4}-Y_{500}$ correlation is close to $\sim 2$, consistent with that found by Basu 

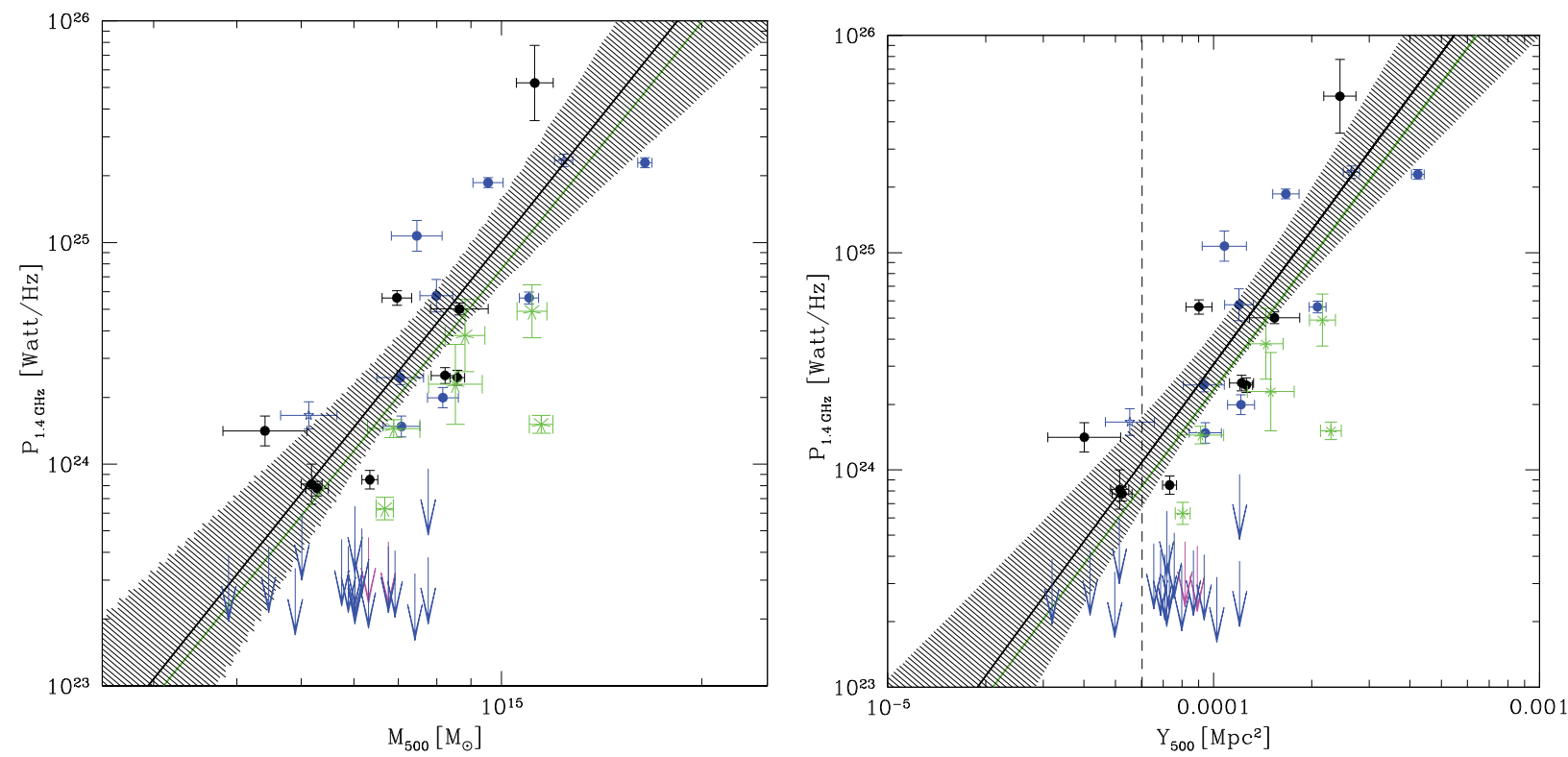

Figure 3. Distribution of clusters in the $P_{1.4}-M_{500}$ (left panel) and in the $P_{1.4}-Y_{500}$ diagrams (right panel). In both panels, the different symbols are as in Figure 2 . Best-fit relations to giant RHs only (black lines) and to all RHs (including USSRH, green dashed lines) are reported. The dashed line in the right panel marks the value $Y_{500}=6 \times 10^{-5} \mathrm{Mpc}^{2}$.

(A color version of this figure is available in the online journal.)

(2012); it is $2.05 \pm 0.28$ when the BCES-bisector method is used, and $2.28 \pm 0.35$ when the BCES-orthogonal method is adopted. The slope of the $P_{1.4}-M_{500}$ correlation is $3.77 \pm 0.57$ and $4.51 \pm 0.78$ in the case of the BCES-bisector and BCESorthogonal methods, respectively, both steeper than previous estimates because of the different definitions of the cluster masses (within a fixed size of $3 \mathrm{Mpc}$, Feretti 2003; or the virial mass, Cassano et al. 2006).

At variance with Basu (2012), we find a clear bimodal behavior of clusters in both diagrams. For $M_{500} \gtrsim 5.5 \times 10^{14} M_{\odot}$ and for $Y_{500} \gtrsim 6 \times 10^{-5} \mathrm{Mpc}^{2}$, all clusters with radio upper limits are well below the $95 \%$ confidence region of the best-fit correlations. For the sake of completeness, for the $P_{1.4}-Y_{\mathrm{SZ}}$ relation, we also performed a regression analysis by making use of the parametric EM algorithm that also deals with upper limits (see Section 3.6). This allows us to evaluate the effect of the radio upper limits on the best-fit correlation, and thus to test the reliability of the correlation and the presence of a bimodal behavior in the cluster radio powers. The best-fit values are reported in Table 3 and the best-fit correlations obtained for giant RHs only and for giant RHs plus upper limits are shown in Figure 4 (solid and dashed line, respectively) together with the 95\% confidence region of the RH-only correlation. All upper limits (with just one exception) lie below the $95 \%$ confidence region, and the two best-fit relations obtained by considering $\mathrm{RHs}$ plus upper limits or only RHs differ both in slope and in normalization.

Our statistical analysis suggests two distinct populations of clusters: those with giant RHs, occupying the region of the correlation, and those without giant RHs, separated from that region.

\subsection{Non-detected Planck Clusters in the $P_{1.4}-Y_{500}$}

With the aim of evaluating the possible position, in the $P_{1.4}-Y_{500}$ diagram, of EGRHS clusters not contained in the 15.5 month PSZ validation catalog, we make use of the correlation between $Y_{500}$ and the core-excluded X-ray luminosity,

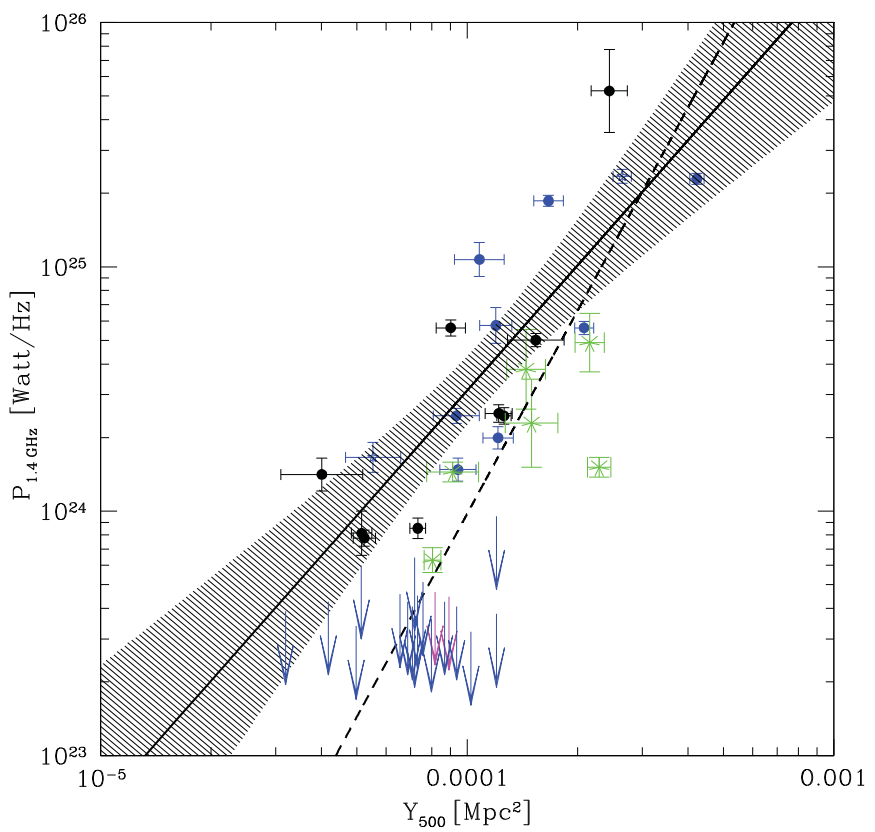

Figure 4. Distribution of clusters in $P_{14}-Y_{500}$ plane. The symbols are as in Figure 2. Best-fit relations to giant RHs (black solid line) and to giant RHs plus upper limits (dashed line) are also shown. The shadowed region show the $95 \%$ confidence region of the best-fit correlation for giant RHs.

(A color version of this figure is available in the online journal.)

$L_{500, \text { nc }}$ (Section 3.2). By using the Planck-XMM-Newton archive sample, which comprises 62 clusters with the highest quality X-ray and SZ data set currently available (Planck Collaboration et al. 2011b), we derive the $0.1-2.4 \mathrm{keV}$ X-ray luminosity between $[0.15-1] R_{500}\left(L_{500, \mathrm{nc}}\right.$, hereafter $)$ and obtained the following $Y_{500}-L_{500, \text { nc }}$ correlation:

$$
h(z)^{-2 / 3} Y_{500}=A\left(\frac{h(z)^{-7 / 3} L_{500, \mathrm{nc}}}{7 \times 10^{44} \mathrm{erg} \mathrm{s}^{-1}}\right)^{B} \mathrm{Mpc}^{2},
$$




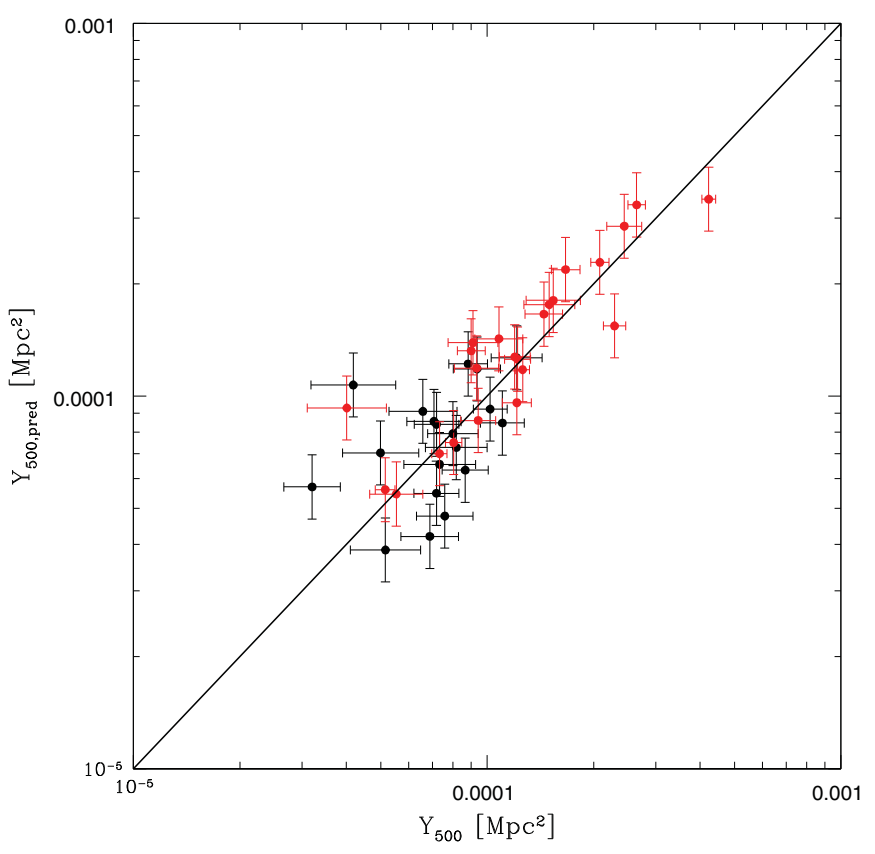

Figure 5. Comparison between the observed values of $Y_{500}$ (in abscissa) and

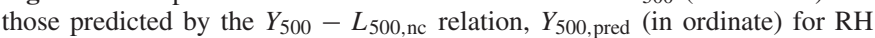
clusters (red points), and clusters with radio upper limits (black points). The black solid line shows the one-to-one trend.

(A color version of this figure is available in the online journal.)

where $h(z)=\sqrt{\Omega_{m}(1+z)^{3}+\Omega_{\Lambda}}, A=10^{-3.795 \pm 0.014}$, and $B=1.094 \pm 0.039$. We thus derive $L_{500, \mathrm{nc}}$ for all clusters in Table $1^{21}$ and then apply Equation (15) to estimate their $Y_{500}$ parameters. To test the consistency of this approach, we compare the "observed" and "predicted" values of $Y_{500}$ for the clusters present in the PSZ catalog. Such a comparison is shown in Figure 5; the data are consistent with a one-to-one trend (with increasing scatter at lower values), suggesting that indeed we can apply this procedure to get reliable estimates of the $Y_{500}$ for clusters not contained in the PSZ catalog.

In Figure 6, we show the distribution of all clusters in the $L_{1.4}-Y_{500}$ diagram, including those that are actually not observed by Planck (dashed arrows). As expected, the bulk of clusters missing in the PSZ catalog is in the region of lower $Y_{500}$ values and with $M_{500}<5.5 \times 10^{14} M_{\odot}$, where the PSZ catalog is only marginally complete (the completeness is $\sim 20 \%$ ). There are, however, two exceptions : RXCJ1532.9+3021, a luminous cool core cluster, and RXCJ0437.1+0043, which are expected in the region of massive clusters.

\subsection{On the $P_{1.4}-Y_{500}$ Scaling Relation}

If we focus on clusters belonging to the EGRHS and also consider the two clusters (A1995 and the Bullet cluster) which are in the same X-ray luminosity and redshift range of the EGRHS clusters, ${ }^{22}$ we find a segregation of clusters in the $P_{1.4}-Y_{500}$ diagram for $Y_{500} \gtrsim 6 \times 10^{-5} \mathrm{Mpc}^{2}$ : clusters with RHs follow a trend between their radio power and the cluster SZ parameter, while clusters without RHs populate the region of radio upper limits, which is a factor of $\sim 5-7$ below

${ }^{21}$ With the exceptions of Coma, MACS1149.5+2223, and PLK171.9-40.7, for which the $L_{500}$ were taken from the literature (see Table 1).

22 A1995 belongs to the NORAS survey which has a slightly lower flux limit with respect to the eBCS used to select the GRHS; the Bullet cluster is in the south and not easily accessible for the GMRT.

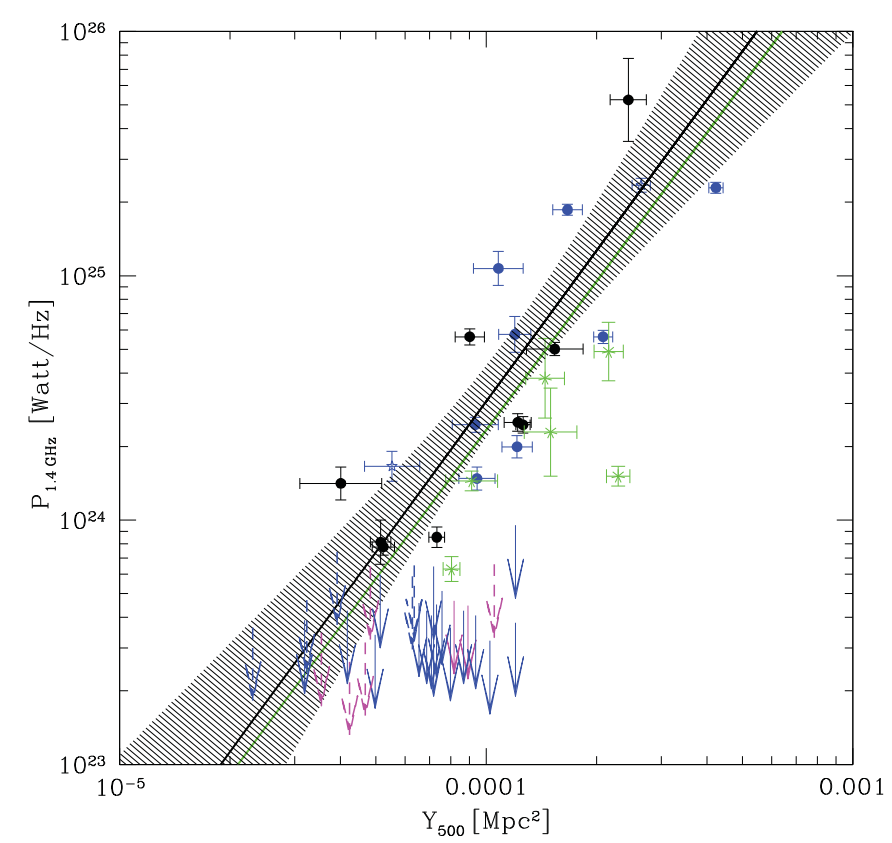

Figure 6. Distribution of clusters in the plane $P_{1.4}-Y_{500}$. The symbols are as in Figure 2, with the dashed arrows indicating the predicted positions of clusters currently not present in the Planck catalog. The best-fit to giant RHs only (black solid line) and to giant RHs plus USSRH (green line) are also shown. The shadowed region show the $95 \%$ confidence region of the best-fit correlation for giant RHs only.

(A color version of this figure is available in the online journal.)

the correlation (Figure 3, right panel). On the other hand, for $Y_{500} \lesssim 6 \times 10^{-5} \mathrm{Mpc}^{2}$, upper limits are not deep enough and lie within the $95 \%$ confident region of the best-fit correlation.

In order to better understand this behavior of clusters and shed light on the mechanism responsible for the formation of giant RHs in clusters, we looked at the dynamical properties of clusters in the $P_{1.4}-Y_{500}$ diagram, adopting the centroid shift variance, $w$, and the surface brightness concentration parameter, $c$, to differentiate between merging $(w<0.012$ and $c>0.2)$ and more relaxed $(w>0.012$ and $c<0.2)$ systems (see Section 3.5).

For $Y_{500} \lesssim 6 \times 10^{-5} \mathrm{Mpc}^{2}$, we find that four clusters with radio upper limits detected by Planck are merging clusters (Figure 7, left panel). Unfortunately, the $L_{1.4}-Y_{500}$ correlation predicts an $\mathrm{RH}$ power for those clusters that is below the sensitivity of the current radio data. In light of our results, the apparent lack of a giant $\mathrm{RH}$ in the merging but relatively low-mass cluster A2146 (Russell et al. 2011) is not surprising, because even if a halo is present in this cluster, it may not be luminous enough to be detected. The only RH cluster with $Y_{500} \lesssim 6 \times 10^{-5} \mathrm{Mpc}^{2}$ is A1995, which in Figure 7 (left panel) is located in a region generally populated by "relaxed" clusters. However, A1995 is a merging system, but the merger is happening mainly along the line of sight (Boschin et al. 2012), and for this reason, its position in the $c-w$ diagram is likely biased by projection effects.

Clusters with $Y_{500} \gtrsim 6 \times 10^{-5} \mathrm{Mpc}^{2}$ show a clear segregation in their dynamical properties. All clusters with detected giant RHs are clearly merging systems, while the majority of clusters with upper limits $(\sim 80 \%)$ are more relaxed (Figure 7 , right panel). The presence of a segregation in the dynamical state of clusters with detected and non-detected $\mathrm{RH}$ strengthens the separation of clusters in the $P_{1.4}-Y_{500}$ diagram and suggests 



Figure 7. Distribution of clusters in the plane $c-w$. Clusters with $Y_{500} \lesssim 6 \times 10^{-5} \mathrm{Mpc}^{2}$ (left panel) and clusters with $Y_{500} \gtrsim 6 \times 10^{-5} \mathrm{Mpc}^{2}$ (right panel) are reported. The black open points are clusters with radio upper limits, while clusters with giant RH and with USSRH are shown as the red points and green asterisks, respectively. The vertical and horizontal dashed lines: $c=0.2$ and $w=0.012$.

(A color version of this figure is available in the online journal.)

that mergers have a crucial role in the formation of these clusterwide diffuse radio sources.

Another interesting observation is that all clusters with $Y_{500}>$ $1.3 \times 10^{-4} \mathrm{Mpc}^{2}$ are merging clusters and host a giant RH. These clusters are very massive systems with $M_{500} \gtrsim 8 \times 10^{14} M_{\odot}$. In particular, if we consider only clusters belonging to the EGRHS (plus the "Bullet" cluster lying within the same redshift range), we have six clusters with $Y_{500}>1.3 \times 10^{-4} \mathrm{Mpc}^{2}$ : four giant RHs and two USSRH. Why do we not find massive relaxed clusters in the EGRHS? The EGRHS is an X-ray selected sample, thus there are no reasons why we should miss a population of massive relaxed clusters, which are generally $\mathrm{X}$-ray luminous. A possibility is that the $Y_{500}$ estimates for merging clusters are biased high with respect to $M_{500}$. Numerical simulations show that merging clusters fall below the $M-Y$ scaling relation, such that their inferred masses could be biased low (e.g., Krause et al. 2012). However, recent observations based on SZ and weak-lensing cluster mass measurements show that merging clusters have weak-lensing masses $40 \%$ lower than relaxed clusters at fixed $Y_{500}$, so that their inferred SZ masses are biased high (e.g., Marrone et al. 2012). The latter authors suggested that the possible cause of these discrepancies could be found in the over-simplicity of the adopted models to fit the weak-lensing data.

A more promising hypothesis is that the lack of massive relaxed systems in the EGRHS is due to the redshift range of this sample, $z \simeq 0.2-0.4$, which is not far from the formation epoch of these massive systems, $M_{500} \gtrsim 8 \times 10^{14} M_{\odot}$ (e.g., Giocoli et al. 2007, 2012). In this case, the probability to observe massive relaxed clusters is smaller; we will investigate these points in more detail in a separate paper (R. Cassano et al., in preparation).

\section{SUMMARY AND CONCLUSIONS}

A number of correlations between thermal and non-thermal cluster properties, i.e., $P_{1.4}-L_{X}, P_{1.4}-M$, and $P_{1.4}-T_{X}$, have been reported for clusters hosting giant RHs since the last decade. However, due to the small statistics and to the lack of statistical samples of clusters observed at radio wavelengths, the reliability of these correlations and the effects of observational biases were not clear (e.g., Rudnick et al. 2006). Only recently, thanks to the GRHS (Venturi et al. 2007, 2008), it has been possible to rely upon a solid sample of clusters with homogeneous and deep radio observations. For the first time, it was possible to place firm upper limits to the diffuse radio flux of clusters without extended diffuse radio emission at the detection level of the survey. These upper limits allowed for the study of the distribution of clusters in the $P_{1.4}-L_{X}$ and to discover a bimodal behavior in the population of clusters: $\mathrm{RH}$ clusters lying on the $P_{1.4}-L_{X}$ correlation and radio-quiet clusters (Brunetti et al. 2007, 2009). Most important, the separation between RH and radio-quiet clusters has a correspondence in the dynamical state of clusters, with merging systems that harbor RHs and radio-quiet clusters that are statistically more relaxed (Cassano et al. 2010). The bimodality has been questioned in the light of the cross-correlation of the GRHS with the Planck ESZ cluster catalog. It was shown that while almost all RHs have been detected in SZ, only 4 out of 20 upper limits were detected (Basu 2012). This was interpreted as a weaker or absent bimodality in the radio-SZ plane. The proposed explanation for this was that SZ measurements allow an unbiased estimate of the cluster mass, whereas X-ray based cluster samples are biased towards the detection of bright cool core clusters, which may induce an apparent bimodal distribution of clusters in the radio-X-ray plane (Basu 2012).

In this paper, we revise the radio-X-ray and radio-SZ correlations. Our analysis is based on the EGRHS (Kale et al. 2013). We searched and found information in the ROSAT and Chandra archive for a subsample of 40 clusters: 29 with upper limits to the radio powers and 11 with giant RHs. In addition to this sample, we also found information for a sample of 14 clusters hosting well-known RHs from the literature. These are 
used to obtain better leverage in radio/X-ray luminosities, which helps in the derivation of more robust scaling relations.

First, we derive the correlation between the monochromatic radio power of halos at $1.4 \mathrm{GHz}$ and the $0.1-2.4 \mathrm{keV}$ band $\mathrm{X}$-ray luminosity of the parent cluster. We revaluate in a homogeneous way the radio flux of all the halos by using GMRT and literature data and measure the X-ray luminosity of the clusters within $R_{500}$ from pointed ROSAT observations and Chandra when ROSAT data are not available (or not sensitive enough). For the first time, we show the presence of a scaling $P_{1.4 \mathrm{GHz}} \propto L_{500}^{2.1 \pm 0.2}$. Being steeper than other halos, USSRH are in general under-luminous with respect to this correlation. Their inclusion in the fit procedure produces a slightly lower normalization and an increase of the scatter with respect to the best-fit relation. We also correct the X-ray luminosity of the parent cluster by modeling the X-ray brightness distribution and excising the cool core. We find that for $L_{500}$ (or $L_{500, \text { cor }}$ ) $\gtrsim 5 \times 10^{44} \mathrm{erg} \mathrm{s}^{-1}$, the distribution of clusters in the $\left(P_{1.4 \mathrm{GHz}}, L_{500}\right)$ and $\left(P_{1.4 \mathrm{GHz}}, L_{500, \text { cor }}\right)$ planes is bimodal: $\mathrm{RH}$ clusters lie on the correlation, while clusters with upper limits to the radio power are below the $95 \%$ confidence region of the best-fit correlation. This allows us to conclude that the presence of cool-core clusters does not affect the bimodal behavior of clusters in the radio power X-ray luminosity plane.

To investigate the behavior of clusters in the radio-SZ diagram, we cross-checked the sample of clusters selected from the EGRHS with the 15.5 month PSZ catalog (Planck Collaboration et al. 2013b) and found SZ information for all 11 RHs and for 19 out of 29 clusters with upper limits. Also, for the remaining 14 clusters with giant RHs, we found information in the PSZ catalog. We found a clear correlation between the RH $P_{1.4}$ and the cluster $Y_{500}$ of the form $P_{1.4} \propto Y_{500}^{2.05 \pm 0.28}$, in line with previous findings (Basu 2012). However, contrary to previous findings, at least for $Y_{500} \gtrsim 6 \times 10^{-5} \mathrm{Mpc}^{2}$ (roughly corresponding to $M_{500} \gtrsim 5.5 \times 10^{14} M_{\odot}$ ), we find that all clusters with radio upper limits lie below the $95 \%$ confidence region of the best-fit correlation, highlighting a bimodal behavior of clusters in the radio-SZ diagram. This segregation is strengthened by the separation of those clusters in the morphological diagrams: clusters with diffuse radio emission are merging clusters, while the great majority of clusters with upper limits are relaxed, thus highlighting the importance of merging events in the generation of giant RHs. We also use the tight correlation between the core-excised cluster $\mathrm{X}$-ray luminosity $L_{500, \text { nc }}$ and $Y_{500}$ to derive the predicted value of $Y_{500}$ for those clusters in our sample that are actually not detected by Planck. As expected, we found that the majority of them (8 out of 10) are in clusters with $Y_{500} \lesssim 6 \times 10^{-5} \mathrm{Mpc}^{2}$, where the completeness of the PSZ catalog is poor (about $20 \%$ ). Interestingly, half of the non-detected clusters are cool core clusters; only two of seven cool core clusters of our sample were detected by Planck, suggesting that in the region of lower completeness, Planck loses preferentially cool-core clusters with respect to merging systems.

The EGRHS is not selected in mass but in X-ray luminosity. However, considering that the completeness of the PSZ catalog for $M_{500} \geqslant 6 \times 10^{14} M_{\odot}$ at $0.2 \leqslant z \leqslant 0.33$ is $\sim 80 \%$ (Planck Collaboration et al. 2013b) and cross-correlating the PSZ catalog with the EGRHS, we estimated that the completeness in mass of the EGRHS is $\sim 55 \%$, and the addition of radio observations of $\sim 17$ galaxy clusters from the PSZ catalog will provide a sample of mass selected clusters with deep radio data and a completeness of $\sim 80 \%$. For a comparison, assuming the same masses and redshift range, we estimated that the completeness of the ESZ Planck catalog is of the order of $35 \%$.

Remarkably, we found that for $Y_{500} \gtrsim 1.3 \times 10^{-4} \mathrm{Mpc}^{2}$ (or $M_{500} \gtrsim 8 \times 10^{14} M_{\odot}$ ), all clusters of the EGRHS are in the process of merging and have a RH. We consider several possibilities to explain this result and conclude that the most likely explanation is that we are looking at these massive systems near their formation epoch (we selected clusters at $z \sim 0.2-0.4$ ), and thus the probability to observe massive relaxed systems at these redshift should be relatively low.

In Section 4, we derive basic scaling relations predicted by the two main scenarios put forward so far to explain giant RH. Under the assumption that synchrotron emission dominates energy losses of relativistic electrons in the ICM, and that the ratio between the energy density of cosmic-ray protons and thermal ICM in the radio emitting volume does not depend on cluster mass, "secondary models" predict that the synchrotron power of the halos scales as $v P^{\text {syn }} \propto L_{X}^{1.6-1.5}$ (e.g., Kushnir et al. 2009) and $v P^{\text {syn }} \propto Y_{500}^{1.55-1.43}$. These scalings are flatter than those derived from observations in the present paper (see Table 3). Re-acceleration models typically predict steeper slopes. For example, under similar assumptions for magnetic field and cosmic rays, following Cassano et al. (2007), the scalings of the halo radio power with the cluster mass and SZ flux are $v P^{\text {syn }} \propto M_{500}^{4}$ and $v P^{\text {syn }} \propto Y_{500}^{2.3}$, respectively, and are in agreement with the observed scalings (see Table 3). A detailed comparison between model expectations and observed scalings which considers the full range of model parameters is beyond the aim of this paper.

It is also worth mentioning that in both the radio-X-ray and radio-SZ diagrams, clusters with USSRH are all below the $95 \%$ confidence region of the best-fit correlations. They are preferentially located in the region between "classical" RHs and radio upper limits. This is not surprising, since these RHs are steeper than those on the correlations, and thus their synchrotron emissivity at $1.4 \mathrm{GHz}$ is lower with respect to that of $\mathrm{RHs}$ with flatter spectra. Interestingly, their position relative to the correlations was already predicted by models in which RHs are generated as a result of the turbulent re-acceleration of relativistic electrons in the ICM (e.g., Cassano 2010; Donnert et al. 2013).

We thank the referee for the useful comments. R.C. thanks Nemmen Rodrigo for providing the patched BCES routine running with gfortran in Mac, and N. Aghanim, D. Dallacasa, J. Donnert, C. Giocoli, P. Mazzotta, and F. Vazza, for useful discussions. We thank H. Bourdin for supplying additional data. K.D. acknowledges the support by the DFG Cluster of Excellence "Origin and Structure of the Universe." S.G. acknowledges the support of NASA through Einstein Postdoctoral Fellowship PF0-110071 awarded by the Chandra X-ray Center (CXC), which is operated by SAO.

\section{REFERENCES}

Ackermann, M., Ajello, M., Allafort, A., et al. 2010, ApJL, 717, L71

Akritas, M. G., \& Bershady, M. A. 1996, ApJ, 470, 706

Arnaud, M., \& Evrard, A. E. 1999, MNRAS, 305, 631

Arnaud, M., Pointecouteau, E., \& Pratt, G. W. 2005, A\&A, 441, 893

Arnaud, M., Pratt, G. W., Piffaretti, R., et al. 2010, A\&A, 517, A92 Basu, K. 2012, MNRAS, 421, L112

Biffi, V., Dolag, K., \& Böhringer, H. 2013, MNRAS, 428, 1395

Böhringer, H., Pratt, G. W., Arnaud, M., et al. 2010, A\&A, 514, 32

Böhringer, H., Schuecker, P., Guzzo, L., et al. 2004, A\&A, 425, 367

Bonafede, A., Brüggen, M., van Weeren, R., et al. 2012, MNRAS, 426, 40 
Bonafede, A., Feretti, L., Giovannini, G., et al. 2009, A\&A, 503, 707 Boschin, W., Girardi, M., \& Barrena, R. 2012, A\&A, 547, A44 Brown, S., Emerick, A., Rudnick, L., \& Brunetti, G. 2011, ApJL, 740, L28

Brunetti, G., Blasi, P., Reimer, O., et al. 2012, MNRAS, 426, 956

Brunetti, G., Cassano, R., Dolag, K., \& Setti, G. 2009, A\&A, 507, 661

Brunetti, G., Giacintucci, S., Cassano, R., et al. 2008, Natur, 455, 944

Brunetti, G., Setti, G., Feretti, L., \& Giovannini, G. 2001, MNRAS, 320, 365

Brunetti, G., Venturi, T., Dallacasa, D., et al. 2007, ApJL, 670, L5

Buote, D. A. 2001, ApJL, 553, L15

Cassano, R. 2009, in ASP Conf. Ser. 407, The Low Frequency Radio Universe, ed. D. J. Saikia, D. Green, Y. Gupta, \& T. Venturi (San Francisco, CA: ASP), 223

Cassano, R. 2010, A\&A, 517, 10

Cassano, R., Brunetti, G., \& Setti, G. 2006, MNRAS, 369, 1577

Cassano, R., Brunetti, G., Setti, G., et al. 2007, MNRAS, 378, 1565

Cassano, R., Brunetti, G., Venturi, T., et al. 2008, A\&A, 480, 687

Cassano, R., Ettori, S., Giacintucci, S., et al. 2010, ApJL, 721, L82

Cavagnolo, K. W., Donahue, M., Voit, G. M., \& Sun, M. 2009, ApJS, 182, 12

Clarke, T. E., \& Ensslin, T. A. 2006, AJ, 131, 2900

Colafrancesco, S. 1999, Diffuse Thermal and Relativistic Plasma in Galaxy Clusters (Garching, Germany: Max-Planck-Institut fur Extraterrestrische Physik), 269

Condon, J. J., Cotton, W. D., Greisen, E. W., et al. 1998, AJ, 115, 1693

Dallacasa, D., Brunetti, G., Giacintucci, S., et al. 2009, ApJ, 699, 1288

Dennison, B. 1980, ApJL, 239, L93

Dolag, K. 2006, AN, 327, 575

Donnert, J., Dolag, K., Brunetti, G., \& Cassano, R. 2013, MNRAS, 429, 3564

Ebeling, H., Edge, A. C., Allen, S. W., et al. 2000, MNRAS, 318, 333

Ebeling, H., Edge, A. C., Böhringer, H., et al. 1998, MNRAS, 301, 881

Enßlin, T. A., \& Röttgering, H. 2002, A\&A, 396, 83

Farnsworth, D., Rudnick, L., Brown, S., \& Brunetti, G. 2013, ApJ, submitted

Feretti, L. 2002, in IAU Symp. 199, The Universe at Low Radio Frequencies, ed. A. Pramesh Rao, G. Swarup, \& Gopal-Krisna (San Francisco, CA: ASP), 133

Feretti, L. 2003, in ASP Conf. Ser. 301, Matter and Energy in Clusters of Galaxies, ed. S. Bowyer \& C.-Y. Hwang (San Francisco, CA: ASP), 143

Feretti, L., Giovannini, G., Govoni, F., \& Murgia, M. 2012, A\&ARv, 20, 54

Ferrari, C., Govoni, F., Schindler, S., Bykov, A. M., \& Rephaeli, Y. 2008, SSRv, 134,93

Giacintucci, S., Dallacasa, D., Venturi, T., et al. 2011, A\&A, 534, A57

Giacintucci, S., Kale, R., Wik, D. R., Venturi, T., \& Markevitch, M. 2013, ApJ, 766, 18

Giacintucci, S., Venturi, T., Brunetti, G., et al. 2009, A\&A, 505, 45

Giocoli, C., Moreno, J., Sheth, R. K., \& Tormen, G. 2007, MNRAS, 376, 977

Giocoli, C., Tormen, G., \& Sheth, R. K. 2012, MNRAS, 422, 185

Giovannini, G., Bonafede, A., Feretti, L., et al. 2009, A\&A, 507, 1257

Giovannini, G., Tordi, M., \& Feretti, L. 1999, NewA, 4, 141

Govoni, F., Enßlin, T. A., Feretti, L., \& Giovannini, G. 2001, A\&A, 369, 441

Govoni, F., Markevitch, M., Vikhlinin, A., et al. 2004, ApJ, 605, 695

Govoni, F., Murgia, M., Feretti, L., et al. 2005, A\&A, 430, L5

Isobe, T., Feigelson, E. D., Akritas, M. G., \& Babu, G. J. 1990, ApJ, 364, 104

Isobe, T., Feigelson, E. D., \& Nelson, P. I. 1986, ApJ, 306, 490
Jeltema, T. E., \& Profumo, S. 2011, ApJ, 728, 53

Kale, R., Venturi, T., Giacintucci, S., et al. 2013, A\&A, 557, A99

Kempner, J. C., \& Sarazin, C. L. 2001, ApJ, 548, 639

Kim, K.-T., Kronberg, P. P., Dewdney, P. E., \& Landecker, T. L. 1990, ApJ, 355,29

Krause, E., Pierpaoli, E., Dolag, K., \& Borgani, S. 2012, MNRAS, 419, 1766

Kushnir, D., Katz, B., \& Waxman, E. 2009, JCAP, 09, 024

Liang, H. 1999, in Diffuse Thermal and Relativistic Plasma in Galaxy Clusters, ed. H. Bohringer, L. Feretti, \& P. Schuecker (Garching, Germany: MaxPlanck-Institut fur Extraterrestrische Physik), 33

Liang, H., Hunstead, R. W., Birkinshaw, M., \& Andreani, P. 2000, ApJ, 544,686

Macario, G., Markevitch, M., Giacintucci, S., et al. 2011, ApJ, 728, 82

Macario, G., Venturi, T., Brunetti, G., et al. 2010, A\&A, 517, A43

Markevitch, M. 1998, ApJ, 504, 27

Markevitch, M., \& Vikhlinin, A. 2001, ApJ, 563, 95

Marrone, D. P., Smith, G. P., Okabe, N., et al. 2012, ApJ, 754, 119

Maughan, B. J., Jones, C., Forman, W., \& Van Speybroeck, L. 2008, ApJS, 174,117

Mohr, J. J., Fabricant, D. G., \& Geller, M. J. 1993, ApJ, 413, 492

Motl, P. M., Hallman, E. J., Burns, J. O., \& Norman, M. L. 2005, ApJL, 623, L63

Nagai, D. 2006, ApJ, 650, 538

O’Hara, T. B., Mohr, J. J., Bialek, J. J., \& Evrard, A. E. 2006, ApJ, 639, 64

Petrosian, V. 2001, ApJ, 557, 560

Poole, G. B., Fardal, M. A., Babul, A., et al. 2006, MNRAS, 373, 881

Planck Collaboration, Ade, P. A. R., Aghanim, N., et al. 2011a, A\&A, 536, A8

Planck Collaboration, Ade, P. A. R., Aghanim, N., et al. 2011b, A\&A, 536, A11

Planck Collaboration, Ade, P. A. R., Aghanim, N., et al. 2013a, A\&A, 554, A140

Planck Collaboration, Ade, P. A. R., Aghanim, N., et al. 2013b, arXiv:1303.5089

Planck Collaboration, Aghanim, N., Arnaud, M., et al. 2011c, A\&A, 536, A9

Pratt, G. W., Croston, J. H., Arnaud, M., \& Böhringer, H. 2009, A\&A, 498, 361 Reiprich, T. H., \& Böhringer, H. 2002, ApJ, 567, 716

Rossetti, M., Eckert, D., Cavalleri, B. M., et al. 2011, A\&A, 532, A123

Rudnick, L., Delain, K. M., \& Lemmerman, J. A. 2006, AN, 327, 549

Rudnick, L., \& Lemmerman, J. A. 2009, ApJ, 697, 1341

Russell, H. R., McNamara, B. R., Edge, A. C., et al. 2013, MNRAS, 432, 530

Russell, H. R., van Weeren, R. J., Edge, A. C., et al. 2011, MNRAS, 417, L1

Santos, J. S., Rosati, P., Tozzi, P., et al. 2008, A\&A, 483, 35

Schlickeiser, R., Sievers, A., \& Thiemann, H. 1987, A\&A, 182, 21

Schuecker, P., Böhringer, H., Reiprich, T. H., \& Feretti, L. 2001, A\&A, 378,408

van Weeren, R. J., Röttgering, H. J. A., Brüggen, M., \& Cohen, A. 2009, A\&A, 505,991

Ventimiglia, D. A., Voit, G. M., Donahue, M., \& Ameglio, S. 2008, ApJ, 685,118

Venturi, T., Giacintucci, S., Brunetti, G., et al. 2007, A\&A, 463, 937

Venturi, T., Giacintucci, S., Dallacasa, D., et al. 2008, A\&A, 484, 327

Venturi, T., Giacintucci, S., Dallacasa, D., et al. 2013, A\&A, 551, A24

Zhang, Y.-Y., Finoguenov, A., Böhringer, H., et al. 2007, A\&A, 467, 437 